\title{
Fractional Cauchy Problem with Riemann-Liouville Derivative on Time Scales
}

\author{
Ling $W u$ and Jiang Zhu \\ School of Mathematics and Statistics, Jiangsu Normal University, Xuzhou 221116, China \\ Correspondence should be addressed to Jiang Zhu; jzhuccy@163.com
}

Received 19 July 2013; Revised 22 September 2013; Accepted 22 September 2013

Academic Editor: Juan J. Trujillo

Copyright ( $) 2013 \mathrm{~L}$. Wu and J. Zhu. This is an open access article distributed under the Creative Commons Attribution License, which permits unrestricted use, distribution, and reproduction in any medium, provided the original work is properly cited.

$\nabla$-Laplace transform, fractional $\nabla$-power function, $\nabla$-Mittag-Leffler function, fractional $\nabla$-integrals, and fractional $\nabla$-differential on time scales are defined. Some of their properties are discussed in detail. After then, by using Laplace transform method, the existence of the solution and the dependency of the solution upon the initial value for Cauchy-type problem with the RiemannLiouville fractional $\nabla$-derivative are studied. Also the explicit solutions to homogeneous equations and nonhomogeneous equations are derived by using Laplace transform method.

\section{Introduction}

The subject of fractional calculus (see [1]) has gained considerable popularity and importance during the past three decades or so due mainly to its demonstrated applications in numerous seemingly diverse and widespread fields of science and engineering. It does indeed provide several potentially useful tools for solving differential and integral equations and various other problems involving special functions of mathematical physics as well as their extensions and generalizations in one and more variables.

On the other hand, in real applications, it is not always a continuous case, but also a discrete case. So, an useful tool as that time scale is considered. In order to unify differential equations and difference equations, Higer proposed firstly the time scale and built the relevant basic theories (see [2-4]). Recently, some authors studied fractional calculus on time scales (see [5-7]). Williams [6] gives a definition of fractional integral and derivative on time scales to unify three cases of specific time scales, which improved the results in [5]. Bastos gives definition of fractional $\Delta$-integral and $\Delta$-derivative on time scales in [7]. In [8], the theory of fractional difference equations has been studied in detail. In the light of the above work, we will further study the theory of fractional integral and derivative on general time scales.
From Theorem 3.1.3 in [6], we know that the integer order $\nabla$-integral on time scales is

$$
\begin{aligned}
I_{\nabla, t_{0}}^{m} f(t) & =\int_{t_{0}}^{t} \nabla t_{1} \int_{t_{0}}^{t_{1}} \nabla t_{2} \cdots \int_{t_{0}}^{t_{m-1}} f\left(t_{m}\right) \nabla t_{m} \\
& =\int_{t_{0}}^{t} \widehat{h}_{m-1}(t, \rho(\tau)) f(\tau) \nabla \tau \\
& =\widehat{h}_{m-1}\left(t, t_{0}\right) * f(t),
\end{aligned}
$$

where $*$ is defined in Definition 31. For continuous case, the fractional $\nabla$-integral (see, e.g., [3]) is defined by

$$
\begin{aligned}
I_{a+}^{\alpha} f(x) & =\int_{a}^{x} \frac{(x-t)^{\alpha-1}}{\Gamma(\alpha)} f(t) d t \\
& =\frac{(x-a)^{\alpha-1}}{\Gamma(\alpha)} * f(x) \quad(x>a ; \alpha>0),
\end{aligned}
$$

while for discrete case, the fractional sum (see, e.g., [8]) is defined by

$$
\begin{aligned}
n_{0} \nabla_{n}^{-v} x(n) & =\sum_{r=0}^{n}\left[\begin{array}{c}
v \\
n-r
\end{array}\right] x(r) \\
& =\left[\begin{array}{l}
v \\
n
\end{array}\right] * x(n) \quad(\nu>0) .
\end{aligned}
$$


Thus, we expect that fractional $\nabla$-integral general on time scales can be defined by

$$
I_{\nabla, t_{0}}^{\alpha} f(t)=\widehat{h}_{\alpha-1}\left(t, t_{0}\right) * f(t) .
$$

To do this, the key problem is that how to define generalized $\nabla$-power function $\widehat{h}_{\alpha}\left(t, t_{0}\right)$ on time scales. In [6], Williams, by using axiomatization method, gives a definition of fractional generalized $\nabla$-power function. However, this definition has no specific form, but it only has an abstract expression. On the other hand, we find that some properties of $\nabla$-power function $\widehat{h}_{k}\left(t, t_{0}\right)$ on time scales under the Laplace transform are important to define fractional generalized $\nabla$-power function $\widehat{h}_{\alpha}\left(t, t_{0}\right)$ on time scales. So, in Section 3, we will give a definition of $\nabla$-Laplace transform, fractional generalized $\nabla$ power function $\widehat{h}_{\alpha}\left(t, t_{0}\right)$ on time scales. Then by using these definitions, we define and study the Riemann-Liouville fractional $\nabla$-integral, Riemann-Liouville fractional $\nabla$-derivative, and $\nabla$-Mittag-Leffler function on time scales. In Section 4 , we present some properties of fractional $\nabla$-integral and fractional $\nabla$-differential on time scales. Then, in Section 5, Cauchy-type problem with the Riemann-Liouville fractional $\nabla$-derivative is discussed. In Section 6, for the RiemannLiouville fractional $\nabla$-differential initial value problem, we discuss the dependency of the solution upon the initial value. In Section 7, by applying the Laplace transform method, we derive explicit solutions to homogeneous equations with constant coefficients. In Section 8, we also use the Laplace transform method to find particular solutions of the corresponding nonhomogeneous equations.

\section{Preliminaries}

First, we present some preliminaries about time scales in [2].

Definition 1 (see [2]). A time scale $\mathbb{T}$ is an nonempty closed subset of the real numbers.

Definition 2 (see [2]). For $t \in \mathbb{T}$, we define the forward jump operator $\sigma: \mathbb{T} \rightarrow \mathbb{T}$ by

$$
\sigma(t):=\inf \{s \in \mathbb{T}: s>t\},
$$

while the backward jump operator $\rho: \mathbb{T} \rightarrow \mathbb{T}$ is defined by

$$
\rho(t):=\sup \{s \in \mathbb{T}: s<t\} .
$$

If $\sigma(t)>t$, we say that $t$ is right-scattered, while if $\rho(t)<t$, we say that $t$ is left-scattered. Points that are right-scattered and left-scattered at the same time are called isolated. Also, if $t<$ $\sup \mathbb{T}$ and $\sigma(t)=t$, then $t$ is called right-dense, and if $t>\inf \mathbb{T}$ and $\rho(t)=t$, then $t$ is called left-dense. Finally, the graininess function $v: \mathbb{T} \rightarrow[0, \infty)$ is defined by

$$
\nu(t):=t-\rho(t) .
$$

Definition 3 (see [2]). If $\mathbb{T}$ has a right-scattered minimum $m$, then we define $\mathbb{T}_{k}=\mathbb{T}-\{m\}$; otherwise, $\mathbb{T}_{k}=\mathbb{T}$. Assume that $f: \mathbb{T} \rightarrow \mathbb{R}$ is a function and let $t \in \mathbb{T}_{k}$. Then we define $f^{\nabla}(t)$ to be the number (provided it exists) with the property that, given any $\varepsilon>0$, there is a neighborhood $U$ of $t$ (i.e., $U=$ $(t-\delta, t+\delta) \cap \mathbb{T}$ for some $\delta>0)$ such that

$$
\begin{gathered}
\left|[f(\rho(t))-f(s)]-f^{\nabla}(t)[\rho(t)-s]\right| \\
\leq \varepsilon|\rho(t)-s|, \quad \forall s \in U .
\end{gathered}
$$

We call $f^{\nabla}(t)$ the nabla derivative of $f$ at $t$.

Definition 4 (see [2]). A function $f: \mathbb{T} \rightarrow \mathbb{R}$ is called regulated provided its right-sided limits exist (finite) at all rightdense points in $\mathbb{T}$ and its left-sided limits exist (finite) at all left-dense points in $\mathbb{T}$.

Definition 5 (see [2, page 100]). The generalized polynomials are the functions $\widehat{h}_{k}: \mathbb{T}^{2}:=\mathbb{T} \times \mathbb{T} \rightarrow \mathbb{R}, k \in \mathbb{N}_{0}$, defined recursively as follows: The function $\widehat{h}_{0}$ is

$$
\widehat{h}_{0}(t, s)=1, \quad \forall s, t \in \mathbb{T},
$$

and given $\widehat{h}_{k}$ for $k \in \mathbb{N}_{0}$, the function $\widehat{h}_{k+1}$ is

$$
\widehat{h}_{k+1}(t, s)=\int_{s}^{t} \widehat{h}_{k}(\tau, s) \nabla \tau, \quad \forall s, t \in \mathbb{T} .
$$

Definition 6 (see [3, page 38]). The generalized polynomials are the functions $h_{k}: \mathbb{T}^{2}:=\mathbb{T} \times \mathbb{T} \rightarrow \mathbb{R}, k \in \mathbb{N}_{0}$, defined recursively as follows: The function $h_{0}$ is

$$
h_{0}(t, s)=1, \quad \forall s, t \in \mathbb{T},
$$

and given $h_{k}$ for $k \in \mathbb{N}_{0}$, the function $h_{k+1}$ is

$$
h_{k+1}(t, s)=\int_{s}^{t} h_{k}(\tau, s) \Delta \tau, \quad \forall s, t \in \mathbb{T} .
$$

Theorem 7 (see [2], Taylor's Formula). Let $n \in \mathbb{N}$. Suppose that the function $f$ is such that $f^{\nabla^{n+1}}$ is ld-continuous on $\mathbb{T}_{k^{n+1}}$. Let $\alpha \in \mathbb{T}_{k^{n}}, t \in \mathbb{T}$. Then one has

$$
f(t)=\sum_{k=0}^{n} \widehat{h}_{k}(t, \alpha) f^{\nabla^{k}}(\alpha)+\int_{\alpha}^{t} \widehat{h}_{n}(t, \rho(\tau)) f^{\nabla^{n+1}}(\tau) \nabla \tau .
$$

Definition 8 (see [6]). A subset $I \subset \mathbb{T}$ is called a time scale interval, if it is of the form $I=A \cap \mathbb{\mathbb { T }}$ for some real interval $A \subset \mathbb{R}$. For a time scale interval $I$, a function $f: I \rightarrow \mathbb{R}$ is said to be left dense absolutely continuous if for all $\varepsilon>0$ there exist $\delta>0$ such that $\sum_{k=1}^{n}\left|f\left(b_{k}\right)-f\left(a_{k}\right)\right|<\varepsilon$ whenever a disjoint finite collection of subtime scale intervals $\left(a_{k}, b_{k}\right] \cap$ $\mathbb{T} \subset I$ for $1 \leq k \leq n$ satisfies $\sum_{k=1}^{n}\left|b_{k}-a_{k}\right|<\delta$. We denote $f \in A C$. If $f^{\nabla^{m-1}} \in A C$, then we denote $f \in A C_{\nabla}^{m}$.

According to Theorem 4.13 in [9], we have the following lemma.

Lemma 9. Let $E \subset \mathbb{T}-\{\max \mathbb{T}\}$ be a measurable set. If $f$ : $\mathbb{T} \rightarrow \mathbb{R}$ is integrable on $E$, then

$$
\int_{E} f^{\sigma}(s) \Delta s=\int_{E} f(s) \nabla s .
$$


Proof. From Theorem 4.13 in [9], we have that

$$
\begin{aligned}
\int_{E} f^{\sigma}(s) \Delta s & =\int_{E} f^{\sigma}(s) d s+\sum_{i \in I_{E}} f^{\sigma}\left(t_{i}\right) \mu\left(t_{i}\right) \\
& =\int_{E} f(s) d s+\sum_{i \in I_{E}} f\left(\sigma\left(t_{i}\right)\right) \mu\left(t_{i}\right) \\
& =\int_{E} f(s) \nabla s,
\end{aligned}
$$

where $I_{E}$ denotes the indices set of right-scattered points of $E$.

Definition 10 (see [8]). The increasing factorial function is defined as

$$
(x)^{(n)}=x(x+1)(x+2) \cdots(x+n-1),
$$

where $n$ is a positive integer and $x$ is a real number, and the symbol $\left[\begin{array}{l}x \\ n\end{array}\right]$ is defined as

$$
\left[\begin{array}{l}
x \\
n
\end{array}\right]=\frac{x(x+1)(x+2) \cdots(x+n-1)}{n !} .
$$

Definition 11 (see [8]). Let $p>0$. The fractional sum whose lower limit is $n_{0}$ is defined as

$$
n_{0} \nabla_{n}^{-p} f(n)=\sum_{r=n_{0}}^{n}\left[\begin{array}{c}
p \\
n-r
\end{array}\right] f(r) .
$$

Definition 12 (see [8]). Let $p>0$ and $m=[p]+1$. The fractional difference whose lower limit is $n_{0}$ is defined as

$$
n_{0} \nabla_{n}^{p} f(n)=\nabla^{m}\left[{ }_{n_{0}} \nabla_{n}^{-(m-p)} f(n)\right] .
$$

In our discussion, we also need some information about $\nabla$-exponential function.

Definition 13 (see [2, Definition 3.4]). The function $p$ is $v$ regressive if

$$
1-v(t) p(t) \neq 0
$$

for all $t \in \mathbb{T}_{k}$. Define the $\nu$-regressive class of functions on $\mathbb{T}_{k}$ to be

$$
\mathscr{R}_{v}=\{p: \mathbb{T} \longrightarrow \mathbb{R} \mid p \text { is ld continuous and } \nu \text {-regressive }\} .
$$

For $p \in \mathscr{R}_{v}$, define circle minus $p$ by

$$
\ominus_{\nu} p:=-\frac{p}{1-p \nu}
$$

Definition 14 (see [2, Definition 3.9]). For $h>0$, let

$$
\begin{gathered}
\mathbb{Z}_{h}:=\left\{z \in \mathbb{C}: \frac{-\pi}{h}<\operatorname{Im}(z)<\frac{\pi}{h}\right\}, \\
\mathbb{C}_{h}:=\left\{z \in \mathbb{C}: z \neq \frac{1}{h}\right\} .
\end{gathered}
$$

Define the $v$-cylinder transformation $\widehat{\xi}_{h}: \mathbb{C}_{h} \rightarrow \mathbb{Z}_{h}$ by

$$
\widehat{\xi}_{h}(z):=-\frac{1}{h} \log (1-z h),
$$

where Log is the principal logarithm function.

Definition 15 (see [2, Definition 3.10]). If $p \in \mathscr{R}_{v}$, then one defines the nabla exponential function by

$$
\widehat{e}_{p}(t, s):=\exp \left(\int_{s}^{t} \widehat{\xi}_{v(\tau)}(p(\tau)) \nabla \tau\right), \quad \text { for } s, t \in \mathbb{T}
$$

where the $\nu$-cylinder transformation $\widehat{\xi}_{h}$ is as in (24).

Definition 16 (see [2, Definition 3.12]). If $p \in \mathscr{R}_{v}$, then the first-order linear dynamic equation

$$
y^{\nabla}=p(t) y
$$

is called $\nu$-regressive.

Lemma 17 (see [2, Lemma 3.11]). If $p \in \mathscr{R}_{v}$, then the semigroup property

$$
\widehat{e}_{p}(t, u) \widehat{e}_{p}(u, s)=\widehat{e}_{p}(t, s), \quad \forall u, s, t \in \mathbb{T}
$$

is satisfied.

Theorem 18 (see [2, Theorem 3.13]). Suppose that (26) is $v$ regressive and fix $t_{0} \in \mathbb{T}$. Then $\widehat{e}_{p}\left(\cdot, t_{0}\right)$ is a solution of the initial value problem

$$
y^{\nabla}=p(t) y, \quad y\left(t_{0}\right)=1
$$

on $\mathbb{T}$.

Theorem 19 (see [2, Theorem 3.15(ii)]). If $p \in \mathscr{R}_{v}$, then

$$
\widehat{e}_{p}(\rho(t), s):=\widehat{e}_{p}^{\rho}(t, s)=(1-v(t) p(t)) \hat{e}_{p}(t, s) .
$$

\section{3. $\nabla$-Laplace Transform, Fractional Generalized $\nabla$-Power Function, Fractional $\nabla$-Integral and Derivative, and $\nabla$-Mittag-Leffler Function}

In this section, we first define $\nabla$-Laplace transform and discuss the properties of $\nabla$-Laplace transform. By using the inverse $\nabla$-Laplace transform, we define fractional generalized $\nabla$-power function, which is a basis of our definitions of fractional $\nabla$-integral and fractional $\nabla$-derivative.

From now on, we always assume that $t_{0} \in \mathbb{T}$, sup $\mathbb{\mathbb { V }}=\infty$. Note that if we assume that $z \in \mathscr{R}_{\nu}$ is a constant, then $\ominus_{\gamma} z \in$ $\mathscr{R}_{v}$ and $\widehat{e}_{\ominus_{\gamma} z}\left(t, t_{0}\right)$ is well defined. With this in mind we make the following definition.

Definition 20. Assume that $x: \mathbb{T} \rightarrow \mathbb{R}$ is regulated and $t_{0} \in$ $\mathbb{T}$. Then, the Laplace transform of $x$ is defined by

$$
\mathscr{L}_{\nabla, t_{0}}\{x\}(z)=\int_{t_{0}}^{\infty} x(t) \hat{e}_{\ominus_{\nu} z}^{\rho}\left(t, t_{0}\right) \nabla t,
$$

for $z \in \mathscr{D}\{x\}$, where $\mathscr{D}\{x\}$ consists of all complex numbers $z \in \mathscr{R}_{\nu}$ for which the improper integral exists. 
The following result is needed frequently.

Lemma 21. If $z \in \mathbb{C}$ is regressive, then

$$
\widehat{e}_{\ominus_{v} z}^{\rho}\left(t, t_{0}\right)=\frac{\widehat{e}_{\ominus_{v} z}\left(t, t_{0}\right)}{1-\nu(t) z}=-\frac{\ominus_{v} z}{z} \widehat{e}_{\ominus_{v} z}\left(t, t_{0}\right) .
$$

Proof. By Theorem 19, we have

$$
\begin{aligned}
\widehat{e}_{\ominus_{v} z}^{\rho}\left(t, t_{0}\right) & =\left(1-v(t)\left(\ominus_{v} z\right)\right) \widehat{e}_{\ominus_{v} z}\left(t, t_{0}\right) \\
& =\left(1-\frac{v(t)(-z)}{1-v(t) z}\right) \widehat{e}_{\ominus_{v} z}\left(t, t_{0}\right) \\
& =\frac{\widehat{e}_{\ominus_{v} z}\left(t, t_{0}\right)}{1-v(t) z} \\
& =-\frac{\ominus_{v} z}{z} \widehat{e}_{\ominus_{v} z}\left(t, t_{0}\right) .
\end{aligned}
$$

This proves our claim.

We now will use the Lemma 21 to find the Laplace transform of $x(t) \equiv 1$ as follows:

$$
\begin{aligned}
\mathscr{L}_{\nabla, t_{0}}\{1\}(z) & =\int_{t_{0}}^{\infty} 1 \cdot \widehat{e}_{\ominus_{\nu} z}^{\rho}\left(t, t_{0}\right) \nabla t \\
& =-\frac{1}{z} \int_{t_{0}}^{\infty} \ominus_{\nu} z \widehat{e}_{\ominus_{\nu} z}\left(t, t_{0}\right) \nabla t \\
& =-\left.\frac{1}{z} \widehat{e}_{\ominus_{\nu} z}\left(t, t_{0}\right)\right|_{t_{0}} ^{\infty}=\frac{1}{z},
\end{aligned}
$$

for all complex values of $z \in \mathscr{R}_{v}$ such that $\lim _{t \rightarrow \infty} \widehat{e}_{\ominus_{\gamma} z}\left(t, t_{0}\right)$ $=0$ holds. The following two results are derived using integration by parts.

Theorem 22. Assume that $x: \mathbb{T} \rightarrow \mathbb{C}$ is such that $x^{\nabla}$ is regulated. Then,

$$
\mathscr{L}_{\nabla, t_{0}}\left\{x^{\nabla}\right\}(z)=-x\left(t_{0}\right)+z \mathscr{L}_{\nabla, t_{0}}\{x\}(z),
$$

for those regressive $z \in \mathbb{C}$ satisfying

$$
\lim _{t \rightarrow \infty}\left\{x(t) \widehat{e}_{\ominus_{\nu} z}\left(t, t_{0}\right)\right\}=0 .
$$

Proof. Integration by parts and Lemma 21 directly yield

$$
\begin{aligned}
\mathscr{L}_{\nabla, t_{0}}\left\{x^{\nabla}\right\}(z)= & \int_{t_{0}}^{\infty} x^{\nabla}(t) \cdot \widehat{e}_{\ominus_{\gamma} z}^{\rho}\left(t, t_{0}\right) \nabla t \\
= & \left.x(t) \widehat{e}_{\ominus_{\gamma} z}\left(t, t_{0}\right)\right|_{t_{0}} ^{\infty} \\
& -\int_{t_{0}}^{\infty} x(t) \ominus_{\gamma} z \widehat{e}_{\ominus_{\nu} z}\left(t, t_{0}\right) \nabla t \\
= & -x\left(t_{0}\right)+z \int_{t_{0}}^{\infty} x(t) \cdot \widehat{e}_{\ominus_{\nu} z}^{\rho}\left(t, t_{0}\right) \nabla t \\
= & -x\left(t_{0}\right)+z \mathscr{L}_{\nabla, t_{0}}\{x\}(z),
\end{aligned}
$$

provided that (35) holds.
By a similar way, we have

$$
\begin{aligned}
\mathscr{L}_{\nabla, t_{0}}\left\{x^{\nabla \nabla}\right\}(z)= & \int_{t_{0}}^{\infty} x^{\nabla \nabla}(t) \cdot \widehat{e}_{\ominus_{\nu} z}^{\rho}\left(t, t_{0}\right) \nabla t \\
= & \left.x^{\nabla}(t) \widehat{e}_{\ominus_{\nu} z}\left(t, t_{0}\right)\right|_{t_{0}} ^{\infty} \\
& -\int_{t_{0}}^{\infty} x^{\nabla}(t) \ominus_{\nu} z \widehat{e}_{\ominus_{\gamma} z}\left(t, t_{0}\right) \nabla t \\
= & -x^{\nabla}\left(t_{0}\right)+z \int_{t_{0}}^{\infty} x^{\nabla}(t) \cdot \widehat{e}_{\ominus_{\nu} z}^{\rho}\left(t, t_{0}\right) \nabla t \\
= & -x^{\nabla}\left(t_{0}\right)+z \mathscr{L}_{\nabla, t_{0}}\left\{x^{\nabla}\right\}(z) \\
= & -x^{\nabla}\left(t_{0}\right)-z x\left(t_{0}\right)+z^{2} \mathscr{L}_{\nabla, t_{0}}\{x\}(z),
\end{aligned}
$$

provided that $\lim _{t \rightarrow \infty}\left\{x^{\nabla}(t) \widehat{e}_{\Theta_{\gamma} z}\left(t, t_{0}\right)\right\}=0$ and (35) holds and thus we can get the following result by induction:

$$
\mathscr{L}_{\nabla, t_{0}}\left\{x^{\nabla^{k}}\right\}(z)=z^{k} \mathscr{L}_{\nabla, t_{0}}\{x\}(z)-\sum_{i=0}^{k-1} z^{k-i-1} x^{\nabla^{i}}\left(t_{0}\right),
$$

for those regressive $z \in \mathbb{C}$ satisfying $\lim _{t \rightarrow \infty}\left\{x^{\nabla^{i}}(t) \widehat{e}_{\ominus_{,} z}\left(t, t_{0}\right)\right\}$ $=0, i=0,1, \ldots, k-1$.

Theorem 23. Assume that $x: \mathbb{T} \rightarrow \mathbb{R}$ is regulated. If

$$
X(t)=\int_{t_{0}}^{t} x(\tau) \nabla \tau
$$

for $t, t_{0} \in \mathbb{T}$; then

$$
\mathscr{L}_{\nabla, t_{0}}\{X\}(z)=\frac{1}{z} \mathscr{L}_{\nabla, t_{0}}\{x\}(z),
$$

for those regressive $z \in \mathbb{C} /\{0\}$ satisfying

$$
\lim _{t \rightarrow \infty}\left\{\widehat{e}_{\ominus_{y} z}\left(t, t_{0}\right) \int_{t_{0}}^{t} x(\tau) \nabla \tau\right\}=0
$$

Proof. By using integration by parts and Lemma 21, we obtain that

$$
\begin{aligned}
\mathscr{L}_{\nabla, t_{0}}\{X\}(z) \\
=\int_{t_{0}}^{\infty} X(t) \cdot \widehat{e}_{\ominus_{\nu} z}^{\rho}\left(t, t_{0}\right) \nabla t \\
=-\frac{1}{z} \int_{t_{0}}^{\infty} X(t) \ominus_{\nu} z \widehat{e}_{\ominus_{\nu} z}\left(t, t_{0}\right) \nabla t \\
=-\frac{1}{z}\left[\left.X(t) \widehat{e}_{\ominus_{\nu} z}\left(t, t_{0}\right)\right|_{t_{0}} ^{\infty}-\int_{t_{0}}^{\infty} x(t) \widehat{e}_{\ominus_{\nu} z}^{\rho}\left(t, t_{0}\right) \nabla t\right] \\
=\frac{1}{z} \mathscr{L}_{\nabla, t_{0}}\{x\}(z),
\end{aligned}
$$

provided that (41) holds. 
Theorem 24. Assume that $\widehat{h}_{k}\left(t, t_{0}\right), k \in \mathbb{N}_{0}$ are defined as in Definition 5. Then,

$$
\mathscr{L}_{\nabla, t_{0}}\left\{\widehat{h}_{k}\left(\cdot, t_{0}\right)\right\}(z)=\frac{1}{z^{k+1}},
$$

for those regressive $z \in \mathbb{C}$ satisfying

$$
\lim _{t \rightarrow \infty}\left\{\widehat{h}_{k}\left(t, t_{0}\right) \widehat{e}_{\ominus_{\nu} z}\left(t, t_{0}\right)\right\}=0 .
$$

Proof. It follows from (33) that (43) holds for $k=0$. Assume that (43) is valid for $k$, and we will show that it is right for $k+1$. In fact, by using Theorem 23, we have that

$$
\begin{aligned}
\mathscr{L}_{\nabla, t_{0}}\left\{\widehat{h}_{k+1}\left(t, t_{0}\right)\right\}(z) & =\mathscr{L}_{\nabla, t_{0}}\left\{\int_{t_{0}}^{t} \widehat{h}_{k}\left(\tau, t_{0}\right) \nabla \tau\right\}(z) \\
& =\frac{1}{z} \mathscr{L}_{\nabla, t_{0}}\left\{\widehat{h}_{k}\left(\cdot, t_{0}\right)\right\}(z) \\
& =\frac{1}{z} \cdot \frac{1}{z^{k+1}}=\frac{1}{z^{k+2}} .
\end{aligned}
$$

The claim follows by the principle of mathematical induction.

It is similar to the proof of Theorems 1.5 and 1.3 in [10], we get the following uniqueness result about the inverse of Laplace transform and initial value theorem.

Theorem 25 (uniqueness of the inverse). If the functions $f$ : $\mathbb{T} \rightarrow \mathbb{R}$ and $g: \mathbb{T} \rightarrow \mathbb{R}$ have the same Laplace transform, then $f=g$.

Theorem 26 (initial value theorem). Let $f: \mathbb{T} \rightarrow \mathbb{R}$ have generalized Laplace transform $F(z)$. Then, $f\left(t_{0}\right)=\lim _{z \rightarrow \infty} z F(z)$.

By the uniqueness of inverse Laplace transform and fixing $s=t_{0}$, we can define fractional $\nabla$-power function $\widehat{h}_{\alpha}\left(t, t_{0}\right)$.

Definition 27. We define fractional generalized $\nabla$-power function on time scales as follows:

$$
\widehat{h}_{\alpha}\left(t, t_{0}\right)=\mathscr{L}_{\nabla, t_{0}}^{-1}\left\{\frac{1}{z^{\alpha+1}}\right\}(t),
$$

to those suitable regressive $z \in \mathbb{C} \backslash\{0\}$ such that $\mathscr{L}_{\nabla, t_{0}}^{-1}$ exist for $\alpha \in \mathbb{R}, t \geq t_{0}$.

Applying the initial value theorem of Laplace transform, for $\alpha>0$, we have

$$
\widehat{h}_{\alpha}\left(t_{0}, t_{0}\right)=\lim _{z \rightarrow \infty} z \cdot \frac{1}{z^{\alpha+1}}=0 .
$$

In particular, when $\alpha=k \in \mathbb{N}$, it follows from Theorems 24 and 25 , we can know that $\widehat{h}_{k}\left(t, t_{0}\right)$ is usual power function on time scales for $t \geq t_{0}$ defined in Definition 5 .

Example 28. When $\mathbb{T}=\mathbb{R}$, the time scale power functions

$$
\widehat{h}_{\alpha}\left(t, t_{0}\right)=\frac{e^{\alpha \log \left(t-t_{0}\right)}}{\Gamma(\alpha+1)},
$$

provided that $\widehat{h}_{\alpha}\left(t, t_{0}\right)$ makes sense. In fact, it follows from Definition 27 that

$$
\mathscr{L}_{\nabla, t_{0}}\left\{\widehat{h}_{\alpha}\left(t, t_{0}\right)\right\}(z)=\mathscr{L}_{t_{0}}\left\{\widehat{h}_{\alpha}\left(t, t_{0}\right)\right\}(z)=\frac{1}{z^{\alpha+1}} .
$$

On the other hand,

$$
\begin{aligned}
\mathscr{L}_{\nabla, t_{0}}\left\{\frac{e^{\alpha \log \left(t-t_{0}\right)}}{\Gamma(\alpha+1)}\right\}(z) & =\mathscr{L}_{t_{0}}\left\{\frac{\left(t-t_{0}\right)^{\alpha}}{\Gamma(\alpha+1)}\right\}(z) \\
& =\frac{1}{\Gamma(\alpha+1)} \int_{t_{0}}^{\infty}\left(t-t_{0}\right)^{\alpha} e^{-z\left(t-t_{0}\right)} d t \\
& =\frac{1}{\Gamma(\alpha+1)} \cdot \frac{1}{z^{\alpha+1}} \int_{0}^{\infty} u^{\alpha} e^{-u} d u \\
& =\frac{1}{\Gamma(\alpha+1)} \cdot \frac{1}{z^{\alpha+1}} \cdot \Gamma(\alpha+1) \\
& =\frac{1}{z^{\alpha+1}} .
\end{aligned}
$$

Thus, we have that

$$
\begin{aligned}
\mathscr{L}_{t_{0}}\left\{\widehat{h}_{\alpha}\left(t, t_{0}\right)\right\}(z) & =\mathscr{L}_{t_{0}}\left\{\frac{e^{\alpha \log \left(t-t_{0}\right)}}{\Gamma(\alpha+1)}\right\}(z) \\
& =\frac{1}{z^{\alpha+1}} .
\end{aligned}
$$

By using uniqueness of the inverse Laplace transform, we imply that

$$
\widehat{h}_{\alpha}\left(t, t_{0}\right)=\frac{e^{\alpha \log \left(t-t_{0}\right)}}{\Gamma(\alpha+1)} .
$$

Next, in order to define fractional generalized $\nabla$-power function for general $s$, we will present some preliminaries about convolution on time scales. In [11], the definitions of shift and convolution, and some properties about convolution, such as convolution theorem and associativity, are presented for delta case, and in the following, we give them similarly for nabla case.

Let $\mathbb{T}$ be a time scale such that $\sup \mathbb{\mathbb { V }}=\infty$ and fix $t_{0} \in \mathbb{T}$.

Definition 29. For a given $f:\left[t_{0}, \infty\right)_{\mathbb{T}} \rightarrow \mathbb{C}$, the solution of the following shifting problem:

$$
\begin{gathered}
u^{\nabla_{t}}(t, \rho(s))=-u^{\nabla_{s}}(t, s), \quad t, s \in \mathbb{T}, t \geq s \geq t_{0}, \\
u\left(t, t_{0}\right)=f(t), \quad t \in \mathbb{T}, t \geq t_{0},
\end{gathered}
$$

is denoted by $\widetilde{f}$ and is called the shift of $f$.

Example 30. Let $t_{0} \in \mathbb{T}$. Then, for $k \in \mathbb{N}_{0}$,

$$
\widehat{h_{k}\left(\cdot, t_{0}\right)}(t, s)=\widehat{h}_{k}(t, s), \quad t, s \in \mathbb{T} \text {, independent of } t_{0} \text {. }
$$


In fact, it is similar to the discussion for the delta case (refer to [3, page 38$]$ ), and we can prove that

$$
\begin{gathered}
\hat{h}_{k}^{\nabla_{t}}(t, s)=\widehat{h}_{k-1}(t, s), \\
\widehat{h}_{k}^{\nabla_{s}}(t, s)=(-1)^{k} \widehat{g}_{k}^{\nabla_{s}}(s, t)=(-1)^{k} \widehat{g}_{k-1}(s, t)=-\widehat{h}_{k-1}(t, s),
\end{gathered}
$$

where $\widehat{g}_{k}$ is defined by

$$
\begin{gathered}
\widehat{g}_{0}(t, s)=1, \quad \forall s, t \in \mathbb{T}, \\
\widehat{g}_{k+1}(t, s)=\int_{s}^{t} \widehat{g}_{k}(\rho(\tau), s) \nabla \tau, \quad \forall s, t \in \mathbb{T} .
\end{gathered}
$$

Thus, according to Definition 29, we can derive the result.

Definition 31. For given functions $f, g: \mathbb{T} \rightarrow \mathbb{R}$, their convolution $f * g$ is defined by

$$
(f * g)(t)=\int_{t_{0}}^{t} \tilde{f}(t, \rho(\tau)) g(\tau) \nabla \tau, \quad t \in \mathbb{T},
$$

where $\tilde{f}$ is the shift introduced in Definition 29.

Theorem 32 (associativity of the convolution). The convolution is associative; that is,

$$
(f * g) * h=f *(g * h) .
$$

Theorem 33. If $f$ is nabla differentiable, then

$$
(f * g)^{\nabla}=f^{\nabla} * g+f\left(t_{0}\right) g,
$$

and if $g$ is nabla differentiable, then

$$
(f * g)^{\nabla}=f * g^{\nabla}+f g\left(t_{0}\right) .
$$

Theorem 34 (convolution theorem). Suppose that $f, g: \mathbb{T} \rightarrow$ $\mathbb{R}$ are locally $\nabla$-integrable functions on $\mathbb{T}$. Then,

$$
\begin{aligned}
\mathscr{L}_{\nabla}\{f & * g\}(z) \\
& =\mathscr{L}_{\nabla}\{f\}(z) \mathscr{L}_{\nabla}\{g\}(z), \quad z \in \mathscr{D}\{f\} \cap \mathscr{D}\{g\} .
\end{aligned}
$$

In the following, we will define fractional generalized $\nabla$ power function for general $s$.

Definition 35. Fractional generalized $\nabla$-power function $\widehat{h}_{\alpha}(t, s)$ on time scales is defined as the shift of $\widehat{h}_{\alpha}\left(t, t_{0}\right)$, that is

$$
\widehat{h}_{\alpha}(t, s)=\widetilde{h_{\alpha}\left(\cdot, t_{0}\right)}(t, s), \quad\left(t \geq s \geq t_{0}\right) .
$$

According to convolution theorem and Definition 27, we have

$$
\begin{aligned}
\mathscr{L}_{\nabla, t_{0}} & \left\{\widehat{h}_{\alpha}\left(t, t_{0}\right) * \widehat{h}_{\beta}\left(t, t_{0}\right)\right\}(z) \\
& =\mathscr{L}_{\nabla, t_{0}}\left\{\widehat{h}_{\alpha}\left(t, t_{0}\right)\right\}(z) \mathscr{L}_{\nabla, t_{0}}\left\{\widehat{h}_{\beta}\left(t, t_{0}\right)\right\}(z) \\
& =\frac{1}{z^{\alpha+1}} \cdot \frac{1}{z^{\beta+1}} \\
& =\frac{1}{z^{\alpha+\beta+1+1}} \\
& =\mathscr{L}_{\nabla, t_{0}}\left\{\widehat{h}_{\alpha+\beta+1}\left(t, t_{0}\right)\right\}(z) .
\end{aligned}
$$

By the uniqueness of inverse Laplace transform, we obtain

$$
\widehat{h}_{\alpha}\left(t, t_{0}\right) * \widehat{h}_{\beta}\left(t, t_{0}\right)=\widehat{h}_{\alpha+\beta+1}\left(t, t_{0}\right),
$$

that is,

$$
\int_{t_{0}}^{t} \widehat{h}_{\alpha}(t, \rho(\tau)) \widehat{h}_{\beta}\left(\tau, t_{0}\right) \nabla \tau=\widehat{h}_{\alpha+\beta+1}\left(t, t_{0}\right) .
$$

In particular, if $\alpha=0$, then

$$
\int_{t_{0}}^{t} \widehat{h}_{0}(t, \rho(\tau)) \widehat{h}_{\beta}\left(\tau, t_{0}\right) \nabla \tau=\widehat{h}_{\beta+1}\left(t, t_{0}\right),
$$

that is,

$$
\int_{t_{0}}^{t} \widehat{h}_{\beta}\left(\tau, t_{0}\right) \nabla \tau=\widehat{h}_{\beta+1}\left(t, t_{0}\right) .
$$

Thus,

$$
\widehat{h}_{\beta+1}^{\nabla}\left(t, t_{0}\right)=\widehat{h}_{\beta}\left(t, t_{0}\right) \text {. }
$$

If $\beta=0$, then

$$
\int_{t_{0}}^{t} \widehat{h}_{\alpha}(t, \rho(\tau)) \widehat{h}_{0}\left(\tau, t_{0}\right) \nabla \tau=\widehat{h}_{\alpha+1}\left(t, t_{0}\right),
$$

that is,

$$
\int_{t_{0}}^{t} \widehat{h}_{\alpha}(t, \rho(\tau)) \nabla \tau=\widehat{h}_{\alpha+1}\left(t, t_{0}\right) .
$$

According to Theorem 33 and (47), (68), for $\alpha>0$, we have

$$
\begin{aligned}
\left(\widehat{h}_{\alpha}\left(t, t_{0}\right) * g(t)\right)^{\nabla} & =\widehat{h}_{\alpha}^{\nabla}\left(t, t_{0}\right) * g(t)+\widehat{h}_{\alpha}\left(t_{0}, t_{0}\right) * g(t) \\
& =\widehat{h}_{\alpha-1}\left(t, t_{0}\right) * g(t) .
\end{aligned}
$$

Now, we can give definitions of fractional $\nabla$-integral and fractional $\nabla$-derivative on time scales.

From now on, we will always denote $\Omega:=\left[t_{0}, t_{1}\right]_{\mathbb{T}}$ a finite interval on a time scale $\mathbb{T}(\sup \mathbb{T}=\infty)$.

Definition 36. Let $t, t_{0} \in \Omega$. The Riemann-Liouville fractional $\nabla$-integral $I_{\nabla, t_{0}}^{\alpha} f$ of order $\alpha>0$ is defined by

$$
\begin{aligned}
I_{\nabla, t_{0}}^{\alpha} f(t): & =\widehat{h}_{\alpha-1}\left(t, t_{0}\right) * f(t) \\
& \left.=\int_{t_{0}}^{t} \widehat{h_{\alpha-1}\left(\cdot, t_{0}\right.}\right)(t, \rho(\tau)) f(\tau) \nabla \tau \\
& =\int_{t_{0}}^{t} \widehat{h}_{\alpha-1}(t, \rho(\tau)) f(\tau) \nabla \tau \quad\left(t>t_{0}\right) .
\end{aligned}
$$

Definition 37. Let $t, t_{0} \in \Omega$. The Riemann-Liouville fractional $\nabla$-derivative $D_{\nabla, t_{0}}^{\alpha} f$ of order $\alpha \geq 0$ is defined by

$$
D_{\nabla, t_{0}}^{\alpha} f(t)=D_{\nabla}^{m} I_{\nabla, t_{0}}^{m-\alpha} f(t) \quad\left(m=[\alpha]+1 ; t>t_{0}\right) .
$$
$n \in \mathbb{N}$

Throughout this paper, we denote $f^{\nabla^{n}}=D_{\nabla}^{n} f=D_{\nabla, t_{0}}^{n} f$,

In the following, we will give the Laplace transform of fractional $\nabla$-integral and fractional $\nabla$-derivative. 
Lemma 38. Let $\alpha>0, m-1<\alpha \leq m(m \in \mathbb{N})$ and $f: \Omega \rightarrow$ $\mathbb{R}$. For $t_{0}, t \in \Omega_{k^{m}}$ with $t_{0}<t$. Then, we have

(1) if $f \in L_{\nabla, p}(\Omega)$, then

$$
\mathscr{L}_{\nabla, t_{0}}\left\{I_{\nabla, t_{0}}^{\alpha} f(t)\right\}(z)=\frac{1}{z^{\alpha}} \mathscr{L}_{\nabla, t_{0}}\{f(t)\}(z) ;
$$

(2) if $f \in A C_{\nabla}^{m}(\Omega)$, then

$$
\begin{aligned}
\mathscr{L}_{\nabla, t_{0}}\left\{D_{\nabla, t_{0}}^{\alpha} f(t)\right\}(z)= & z^{\alpha} \mathscr{L}_{\nabla, t_{0}}\{f(t)\}(z) \\
& -\sum_{j=1}^{m} z^{j-1} D_{\nabla, t_{0}}^{\alpha-j} f\left(t_{0}\right),
\end{aligned}
$$

for those regressive $z \in \mathbb{C}$ satisfying $\lim _{t \rightarrow \infty}\left\{D_{\nabla}^{j} I_{\nabla, t_{0}}^{m-\alpha} f(t) \widehat{e}_{\ominus_{y} z}\left(t, t_{0}\right)\right\}=0, j=0,1, \ldots, m-1$.

Proof. According to Definition 36, Definition 27 and convolution theorem, we have

$$
\begin{aligned}
\mathscr{L}_{\nabla, t_{0}} & \left\{I_{\nabla, t_{0}}^{\alpha} f(t)\right\}(z) \\
& =\mathscr{L}_{\nabla, t_{0}}\left\{\widehat{h}_{\alpha-1}\left(t, t_{0}\right) * f(t)\right\}(z) \\
& =\mathscr{L}_{\nabla, t_{0}}\left\{\widehat{h}_{\alpha-1}\left(t, t_{0}\right)\right\}(z) \mathscr{L}_{\nabla, t_{0}}\{f(t)\}(z) \\
& =\frac{1}{z^{\alpha}} \mathscr{L}_{\nabla, t_{0}}\{f(t)\}(z) .
\end{aligned}
$$

By Definition 37, (3), and taking the Laplace transform of fractional $\nabla$-integral into account, we get

$$
\begin{aligned}
\mathscr{L}_{\nabla, t_{0}}\left\{D_{\nabla, t_{0}}^{\alpha} f(t)\right\}(z)= & \mathscr{L}_{\nabla, t_{0}}\left\{D_{\nabla}^{m} I_{\nabla, t_{0}}^{m-\alpha} f(t)\right\}(z) \\
= & z^{m} \mathscr{L}_{\nabla, t_{0}}\left\{I_{\nabla, t_{0}}^{m-\alpha} f(t)\right\}(z) \\
& -\sum_{j=0}^{m-1} z^{m-j-1} D_{\nabla}^{j} I_{\nabla, t_{0}}^{m-\alpha} f\left(t_{0}\right) \\
= & z^{m} \frac{1}{z^{m-\alpha}} \mathscr{L}_{\nabla, t_{0}}\{f(t)\}(z) \\
& -\sum_{j=0}^{m-1} z^{m-j-1} D_{\nabla, t_{0}}^{j-m+\alpha} f\left(t_{0}\right) \\
= & z^{\alpha} \mathscr{L}_{\nabla, t_{0}}\{f(t)\}(z) \\
& -\sum_{j=1}^{m} z^{j-1} D_{\nabla, t_{0}}^{\alpha-j} f\left(t_{0}\right),
\end{aligned}
$$

where $D_{\nabla}^{j} I_{\nabla, t_{0}}^{m-\alpha} f\left(t_{0}\right)=D_{\nabla, t_{0}}^{j-m+\alpha} f\left(t_{0}\right)$ follows from the definition of fractional $\nabla$-derivative, and $D_{\nabla, t_{0}}^{\alpha-m} f\left(t_{0}\right)=$ $\lim _{t \rightarrow t_{0}} I_{\nabla, t_{0}}^{m-\alpha} f\left(t_{0}\right)$ ( $t_{0}$ is right-dense); $D_{\nabla, t_{0}}^{\alpha-m} f\left(t_{0}\right)=0\left(t_{0}\right.$ is right scattered).

Finally, we present the definition of $\nabla$-Mittag-Leffler function which is an important tool for solving fractional differential equation. We have known that the Mittag-Leffler function $E_{\alpha}(z):=\sum_{k=0}^{\infty} z^{k} /(\Gamma(\alpha k+1))$ is the fractional order case of exponential function $e^{z}$, and $E_{\alpha, \beta}(z):=\sum_{k=0}^{\infty} z^{k} /(\Gamma(\alpha k$ $+\beta)$ ) is generalized from $E_{\alpha}(z)$. Inspired by these results and exponential function on time scales $\widehat{e}_{\lambda}(t, 0)=\sum_{k=0}^{\infty} \lambda^{k} \widehat{h}_{k}(t, 0)$ (see [7, Remark 124]), we give the following definition.

Definition 39. $\nabla$-Mittag-Leffler function is defined as

$$
{ }_{\nabla} F_{\alpha, \beta}\left(\lambda ; t, t_{0}\right)=\sum_{j=0}^{\infty} \lambda^{j} \widehat{h}_{\alpha j+\beta-1}\left(t, t_{0}\right)
$$

provided that the right hand series is convergent, where $\alpha>$ $0, \lambda, \beta \in \mathbb{R}$.

As to the Laplace transform of $\nabla$-Mittag-Leffler function, we have the following theorem.

Theorem 40. The Laplace transform of $\nabla$-Mittag-Leffler function is

$$
\mathscr{L}_{\nabla, t_{0}}\left\{{ }_{\nabla} F_{\alpha, \beta}\left(\lambda ; t, t_{0}\right)\right\}(z)=\frac{z^{\alpha-\beta}}{z^{\alpha}-\lambda}\left(|\lambda|<|z|^{\alpha}\right) .
$$

Proof. From the definition of Laplace transform, it is obtained that

$$
\begin{aligned}
\mathscr{L}_{\nabla, t_{0}} & \left\{{ }_{\nabla} F_{\alpha, \beta}\left(\lambda ; t, t_{0}\right)\right\}(z) \\
& =\int_{t_{0}}^{\infty} \nabla_{\alpha, \beta}\left(\lambda ; t, t_{0}\right) \cdot \widehat{e}_{\ominus_{\nu} z}^{\rho}\left(t, t_{0}\right) \nabla t \\
& =\int_{t_{0}}^{\infty} \sum_{j=0}^{\infty} \lambda^{j} \widehat{h}_{\alpha j+\beta-1}\left(t, t_{0}\right) \cdot \widehat{e}_{\ominus_{\nu} z}^{\rho}\left(t, t_{0}\right) \nabla t \\
& =\sum_{j=0}^{\infty} \lambda^{j} \int_{t_{0}}^{\infty} \widehat{h}_{\alpha j+\beta-1}\left(t, t_{0}\right) \cdot \widehat{e}_{\ominus_{\nu} z}^{\rho}\left(t, t_{0}\right) \nabla t \\
& =\sum_{j=0}^{\infty} \lambda^{j} \mathscr{L}_{\nabla, t_{0}}\left\{\widehat{h}_{\alpha j+\beta-1}\left(t, t_{0}\right)\right\}(z) \\
& =\sum_{j=0}^{\infty} \lambda^{j} \frac{1}{z^{\alpha j+\beta}} \\
= & z^{-\beta} \sum_{j=0}^{\infty} \lambda^{j}\left(z^{-\alpha}\right)^{j} \\
= & \frac{z^{\alpha-\beta}}{z^{\alpha}-\lambda}\left(|\lambda|<|z|^{\alpha}\right) .
\end{aligned}
$$

By differentiating $k$ times with respect to $\lambda$ on both sides of the formula in the theorem above, we get the following result:

$$
\mathscr{L}_{\nabla, t_{0}}\left\{\frac{\partial^{k}}{\partial \lambda^{k} \nabla} F_{\alpha, \beta}\left(\lambda ; t, t_{0}\right)\right\}(z)=\frac{k ! z^{\alpha-\beta}}{\left(z^{\alpha}-\lambda\right)^{k+1}} .
$$




\section{Properties of Fractional $\nabla$-Integral and Fractional $\nabla$-Derivative}

In this section, we mainly give the properties of fractional $\nabla$ integral and $\nabla$-derivative on time scales which are often used in the following sections.

Property 1. Let $\alpha>0, m=[\alpha]+1, \beta \in \mathbb{R}, t, t_{0} \in \Omega_{k^{m}}$. Then

$$
\begin{aligned}
& \text { (1) } I_{\nabla, t_{0}}^{\alpha} \widehat{h}_{\beta-1}\left(t, t_{0}\right)=\widehat{h}_{\alpha+\beta-1}\left(t, t_{0}\right) \text {; } \\
& \text { (2) } D_{\nabla, t_{0}}^{\alpha} \widehat{h}_{\beta-1}\left(t, t_{0}\right)=\widehat{h}_{\beta-\alpha-1}\left(t, t_{0}\right) .
\end{aligned}
$$

In particular, if $\beta=1, \alpha>0$, then the Riemann-Liouville fractional $\nabla$-derivatives of a constant are, in general, not equal to zero as follows:

$$
D_{\nabla, t_{0}}^{\alpha} 1=\widehat{h}_{-\alpha}\left(t, t_{0}\right), \quad(0<\alpha<1) .
$$

On the other hand, for $j=1,2, \ldots,[\alpha]+1$,

$$
D_{\nabla, t_{0}}^{\alpha} \widehat{h}_{\alpha-j}\left(t, t_{0}\right)=0 \text {. }
$$

In fact,

$$
\begin{aligned}
D_{\nabla, t_{0}}^{\alpha} \widehat{h}_{\alpha-j}\left(t, t_{0}\right) & =D_{\nabla}^{m} I_{\nabla, t_{0}}^{m-\alpha} \widehat{h}_{\alpha-j}\left(t, t_{0}\right) \\
& =D_{\nabla}^{m} \widehat{h}_{m-j}\left(t, t_{0}\right)=0 .
\end{aligned}
$$

Proof. (1) According to Definition 36 and (64), we have

$$
\begin{aligned}
I_{\nabla, t_{0}}^{\alpha} \widehat{h}_{\beta-1}\left(t, t_{0}\right) & =\widehat{h}_{\alpha-1}\left(t, t_{0}\right) * \widehat{h}_{\beta-1}\left(t, t_{0}\right) \\
& =\widehat{h}_{\alpha+\beta-1}\left(t, t_{0}\right) .
\end{aligned}
$$

(2) From Definition 37 and (82), (68), it is obtained that

$$
\begin{aligned}
D_{\nabla, t_{0}}^{\alpha} \widehat{h}_{\beta-1}\left(t, t_{0}\right) & =D_{\nabla}^{m} I_{\nabla, t_{0}}^{m-\alpha} \widehat{h}_{\beta-1}\left(t, t_{0}\right) \\
& =D_{\nabla}^{m} \widehat{h}_{m+\beta-\alpha-1}\left(t, t_{0}\right)=\widehat{h}_{\beta-\alpha-1}\left(t, t_{0}\right) .
\end{aligned}
$$

From Property 1, we derive the following result in [1] when $\mathbb{T}=\mathbb{R}$.

Corollary 41 (see [1]). If $\mathfrak{R}(\alpha) \geq 0$ and $\mathfrak{R}(\beta)>0$, then

$$
\begin{aligned}
& \left(I_{a+}^{\alpha}(t-a)^{\beta-1}\right)(x) \\
& \quad=\frac{\Gamma(\beta)}{\Gamma(\beta+\alpha)}(x-a)^{\beta+\alpha-1}, \quad(\Re(\alpha)>0), \\
& \left(D_{a+}^{\alpha}(t-a)^{\beta-1}\right)(x) \\
& =\frac{\Gamma(\beta)}{\Gamma(\beta-\alpha)}(x-a)^{\beta-\alpha-1}, \quad(\Re(\alpha) \geq 0) .
\end{aligned}
$$

In particular, if $\beta=1$ and $\Re(\alpha) \geq 0$, then the RiemannLiouville fractional derivatives of a constant are, in general, not equal to zero as follows:

$$
\left(D_{a+}^{\alpha} 1\right)(x)=\frac{(x-a)^{-\alpha}}{\Gamma(1-\alpha)}, \quad(0<\Re(\alpha)<1) .
$$

On the other hand, for $j=1,2, \ldots,[\Re(\alpha)]+1$,

$$
\left(D_{a+}^{\alpha}(t-a)^{\alpha-j}\right)(x)=0 .
$$

As to the fractional sum and difference, there is also a similar result in [8].

Corollary 42 (see [8]). Let $p>0, v>0$. Then,

$$
\begin{aligned}
& \text { (1) } n_{0} \nabla_{n}^{-p}\left[\begin{array}{c}
v \\
n-n_{0}
\end{array}\right]=\left[\begin{array}{c}
v+p \\
n-n_{0}
\end{array}\right], \\
& \text { (2) } n_{0} \nabla_{n}^{p}\left[\begin{array}{c}
v \\
n-n_{0}
\end{array}\right]=\left[\begin{array}{c}
v-p \\
n-n_{0}
\end{array}\right] .
\end{aligned}
$$

Property 2. Let $\alpha>0$ and $m=[\alpha]+1, t, t_{0} \in \Omega_{k^{m}}$. If $f(t) \epsilon$ $A C_{\nabla}^{m}(\Omega)$, then the fractional $\nabla$-derivative $D_{\nabla, t_{0}}^{\alpha} f(t)$ which exists almost everywhere on $\Omega_{k^{m}}$ can be represented in the following forms:

$$
\begin{aligned}
D_{\nabla, t_{0}}^{\alpha} f(t)= & \sum_{k=0}^{m-1} \widehat{h}_{k-\alpha}\left(t, t_{0}\right) f^{\nabla^{k}}\left(t_{0}\right) \\
& +\int_{t_{0}}^{t} \widehat{h}_{-\alpha+m-1}(t, \rho(\tau)) f^{\nabla^{m}}(\tau) \nabla \tau .
\end{aligned}
$$

Proof. By Taylor's formula,

$$
\begin{aligned}
f(t)= & \sum_{k=0}^{m-1} \widehat{h}_{k}\left(t, t_{0}\right) f^{\nabla^{k}}\left(t_{0}\right) \\
& +\int_{t_{0}}^{t} \widehat{h}_{m-1}(t, \rho(\tau)) f^{\nabla^{m}}(\tau) \nabla \tau \\
= & \sum_{k=0}^{m-1} \widehat{h}_{k}\left(t, t_{0}\right) f^{\nabla^{k}}\left(t_{0}\right) \\
& +\widehat{h}_{m-1}\left(t, t_{0}\right) * f^{\nabla^{m}}(t)
\end{aligned}
$$

and using (82) and (64), we have

$$
\begin{aligned}
I_{\nabla, t_{0}}^{\alpha} f(t)= & \sum_{k=0}^{m-1} I_{\nabla, t_{0}}^{\alpha} \widehat{h}_{k}\left(t, t_{0}\right) f^{\nabla^{k}}\left(t_{0}\right) \\
& +\widehat{h}_{\alpha-1}\left(t, t_{0}\right) * \widehat{h}_{m-1}\left(t, t_{0}\right) * f^{\nabla^{m}}(t) \\
= & \sum_{k=0}^{m-1} \widehat{h}_{k+\alpha}\left(t, t_{0}\right) f^{\nabla^{k}}\left(t_{0}\right) \\
& +\widehat{h}_{\alpha+m-1}\left(t, t_{0}\right) * f^{\nabla^{m}}(t) \\
= & \sum_{k=0}^{m-1} \widehat{h}_{k+\alpha}\left(t, t_{0}\right) f^{\nabla^{k}}\left(t_{0}\right) \\
& +\int_{t_{0}}^{t} \widehat{h}_{\alpha+m-1}(t, \rho(\tau)) f^{\nabla^{m}}(\tau) \nabla \tau .
\end{aligned}
$$


Besides, according to (95) and taking (68) and (71) into account, we have

$$
\begin{aligned}
D_{\nabla, t_{0}}^{\alpha} f(t)= & D_{\nabla}^{m} I_{\nabla, t_{0}}^{m-\alpha} f(t) \\
= & D_{\nabla}^{m}\left[\sum_{k=0}^{m-1} \widehat{h}_{k+m-\alpha}\left(t, t_{0}\right) f^{\nabla^{k}}\left(t_{0}\right)\right. \\
& \left.+\widehat{h}_{m-\alpha+m-1}\left(t, t_{0}\right) * f^{\nabla^{m}}(t)\right] \\
& =\sum_{k=0}^{m-1} \widehat{h}_{k-\alpha}\left(t, t_{0}\right) f^{\nabla^{k}}\left(t_{0}\right) \\
& +\widehat{h}_{-\alpha+m-1}\left(t, t_{0}\right) * f^{\nabla^{m}}(t) \\
= & \sum_{k=0}^{m-1} \widehat{h}_{k-\alpha}\left(t, t_{0}\right) f^{\nabla^{k}}\left(t_{0}\right) \\
& +\int_{t_{0}}^{t} \widehat{h}_{-\alpha+m-1}(t, \rho(\tau)) f^{\nabla^{m}}(\tau) \nabla \tau .
\end{aligned}
$$

When $\mathbb{T}=\mathbb{R}$, there is the following corollary.

Corollary 43 (see [1]). Let $\mathfrak{R}(\alpha) \geq 0$, and $n=[\Re(\alpha)]+1$. If $y(x) \in A C^{n}[a, b]$, then the fractional derivative $D_{a+}^{\alpha} y$ exists almost everywhere on $[a, b]$ and can be represented in the following form:

$$
\begin{aligned}
\left(D_{a+}^{\alpha} y\right)(x)= & \sum_{k=0}^{n-1} \frac{y^{(k)}(a)}{\Gamma(1+k-\alpha)}(x-a)^{k-\alpha} \\
& +\frac{1}{\Gamma(n-\alpha)} \int_{a}^{x} \frac{y^{(n)}(t) d t}{(x-t)^{\alpha-n+1}} .
\end{aligned}
$$

Similarly, for the fractional sum and difference, there is also the following corollary.

Corollary 44 (see $[8]$ ). For $p>0$ and $m \in \mathbb{N}$, it is valid that

$$
\begin{aligned}
n_{0} \nabla_{n}^{-p} f(n)= & \sum_{k=0}^{m} \nabla^{k} f\left(n_{0}-1\right)\left[\begin{array}{c}
p+k+1 \\
n-n_{0}
\end{array}\right] \\
& +\sum_{r=n_{0}}^{n}\left[\begin{array}{c}
p+m+1 \\
n-r
\end{array}\right] \nabla^{m+1} f(r), \\
n_{0} \nabla_{n}^{p} f(n)= & \sum_{k=0}^{m} \nabla^{k} f\left(n_{0}-1\right)\left[\begin{array}{c}
-p+k+1 \\
n-n_{0}
\end{array}\right] \\
& +\sum_{r=n_{0}}^{n}\left[\begin{array}{c}
-p+m+1 \\
n-r
\end{array}\right] \nabla^{m+1} f(r) .
\end{aligned}
$$

The semigroup property of the fractional $\nabla$-integral operator is given by the following result.
Property 3. If $\alpha>0$ and $\beta>0$, then the equation

$$
I_{\nabla, t_{0}}^{\alpha} I_{\nabla, t_{0}}^{\beta} f(t)=I_{\nabla, t_{0}}^{\alpha+\beta} f(t)
$$

is satisfied at almost every point $t \in \Omega$ for $f(t) \in L_{\nabla, p}(\Omega)(1 \leq$ $p \leq \infty)$.

Proof. According to Definition 36 and (64), and using associativity of the convolution, we have

$$
\begin{aligned}
I_{\nabla, t_{0}}^{\alpha} I_{\nabla, t_{0}}^{\beta} f(t) & =\widehat{h}_{\alpha-1}\left(t, t_{0}\right) * I_{\nabla, t_{0}}^{\beta} f(t) \\
& =\widehat{h}_{\alpha-1}\left(t, t_{0}\right) * \widehat{h}_{\beta-1}\left(t, t_{0}\right) * f(t) \\
& =\widehat{h}_{\alpha+\beta-1}\left(t, t_{0}\right) * f(t) \\
& =I_{\nabla, t_{0}}^{\alpha+\beta} f(t) .
\end{aligned}
$$

The following assertion shows that the fractional differentiation is an operation inverse to the fractional integration from the left.

Property 4. If $\alpha>0$ and $f(t) \in L_{\nabla, p}(\Omega)(1 \leq p \leq \infty)$, then the following equality

$$
D_{\nabla, t_{0}}^{\alpha} I_{\nabla, t_{0}}^{\alpha} f(t)=f(t)
$$

holds almost everywhere on $\Omega$.

Proof. According to the definition of the fractional $\nabla$-derivative and using (99), we get

$$
\begin{aligned}
D_{\nabla, t_{0}}^{\alpha} I_{\nabla, t_{0}}^{\alpha} f(t) & =D_{\nabla}^{m} I_{\nabla, t_{0}}^{m-\alpha} I_{\nabla, t_{0}}^{\alpha} f(t) \\
& =D_{\nabla, t_{0}}^{m} I_{\nabla, t_{0}}^{m} f(t)=f(t) .
\end{aligned}
$$

In the following, we will derive the composition relations between fractional $\nabla$-differentiation and fractional $\nabla$-integration operators.

Property 5. If $\alpha>\beta>0$, then, for $f(t) \in L_{\nabla, p}(\Omega)(1 \leq p \leq$ $\infty)$, the relation

$$
D_{\nabla, t_{0}}^{\beta} I_{\nabla, t_{0}}^{\alpha} f(t)=I_{\nabla, t_{0}}^{\alpha-\beta} f(t)
$$

holds almost everywhere on $\Omega$. In particular, when $\beta=k \in \mathbb{N}$ and $\alpha>k$, then

$$
D_{\nabla, t_{0}}^{k} I_{\nabla, t_{0}}^{\alpha} f(t)=I_{\nabla, t_{0}}^{\alpha-k} f(t)
$$

Proof. The proof is the same with the proof of Property 4, so we omit it. 
Property 6. Let $\alpha>0, m=[\alpha]+1, n \in \mathbb{N}$. If $f(t) \in A C_{\nabla}^{m+n}(\Omega)$, then

(1) $D_{\nabla}^{n} D_{\nabla, t_{0}}^{\alpha} f(t)=D_{\nabla, t_{0}}^{\alpha+n} f(t)$,

(2) $D_{\nabla, t_{0}}^{\alpha} D_{\nabla}^{n} f(t)=D_{\nabla, t_{0}}^{\alpha+n} f(t)$

$$
-\sum_{k=0}^{n-1} \widehat{h}_{-\alpha-n+k}\left(t, t_{0}\right) f^{\nabla^{k}}\left(t_{0}\right) .
$$

Thus,

$$
D_{\nabla, t_{0}}^{\alpha} D_{\nabla}^{n} f(t)=D_{\nabla, t_{0}}^{\alpha+n} f(t)
$$

is valid if and only if

$$
f^{\nabla^{k}}\left(t_{0}\right)=0, \quad k=0, \ldots, n-1 .
$$

Proof. Since $f(t) \in A C_{\nabla}^{m+n}(\Omega)$, by (93) in Property 2 and (71), (68), we have

$$
\begin{aligned}
D_{\nabla}^{n} D_{\nabla, t_{0}}^{\alpha} f(t)= & D_{\nabla}^{n}\left[\sum_{k=0}^{m+n-1} \widehat{h}_{k-\alpha}\left(t, t_{0}\right) f^{\nabla^{k}}\left(t_{0}\right)\right. \\
& \left.+\widehat{h}_{-\alpha+m+n-1}\left(t, t_{0}\right) * f^{\nabla^{m+n}}(t)\right] \\
= & \sum_{k=0}^{m+n-1} \widehat{h}_{k-\alpha-n}\left(t, t_{0}\right) f^{\nabla^{k}}\left(t_{0}\right) \\
& +\widehat{h}_{-\alpha+m-1}\left(t, t_{0}\right) * f^{\nabla^{m+n}}(t) \\
= & D_{\nabla, t_{0}}^{\alpha+n} f(t) .
\end{aligned}
$$

On the other hand, from $f(t) \in A C_{\nabla}^{m+n}(\Omega)$, we know that $D_{\nabla}^{n} f(t) \in A C_{\nabla}^{m}(\Omega)$ and thus, we have

$$
\begin{aligned}
D_{\nabla, t_{0}}^{\alpha} D_{\nabla}^{n} f(t)= & \sum_{k=0}^{m-1} \widehat{h}_{k-\alpha}\left(t, t_{0}\right) f^{\nabla^{k+n}}\left(t_{0}\right) \\
& +\widehat{h}_{-\alpha+m-1}\left(t, t_{0}\right) * f^{\nabla^{m+n}}(t) .
\end{aligned}
$$

Comparing with (108) and (109), we can get that

$$
D_{\nabla, t_{0}}^{\alpha} D_{\nabla}^{n} f(t)=D_{\nabla, t_{0}}^{\alpha+n} f(t)-\sum_{k=0}^{n-1} \widehat{h}_{-\alpha-n+k}\left(t, t_{0}\right) f^{\nabla^{k}}\left(t_{0}\right),
$$

which proves the result.

Property 7. Let $\alpha>0, m=[\alpha]+1, n \in \mathbb{N}$. If $f(t) \in A C_{\nabla}^{n}(\Omega)$, then

$$
\begin{aligned}
I_{\nabla, t_{0}}^{\alpha} D_{\nabla}^{n} f(t)= & D_{\nabla}^{n} I_{\nabla, t_{0}}^{\alpha} f(t) \\
& -\sum_{k=0}^{n-1} \widehat{h}_{\alpha-n-k}\left(t, t_{0}\right) f^{\nabla^{k}}\left(t_{0}\right) .
\end{aligned}
$$

Proof. Applying Laplace transform to $I_{\nabla, t_{0}}^{\alpha} D_{\nabla}^{n} f(t)$, we have

$$
\begin{aligned}
\mathscr{L}_{\nabla, t_{0}} & \left\{I_{\nabla, t_{0}}^{\alpha} D_{\nabla}^{n} f(t)\right\}(z) \\
= & \mathscr{L}_{\nabla, t_{0}}\left\{\widehat{h}_{\alpha-1}\left(t, t_{0}\right) * D_{\nabla}^{n} f(t)\right\}(z) \\
= & \mathscr{L}_{\nabla, t_{0}}\left\{\widehat{h}_{\alpha-1}\left(t, t_{0}\right)\right\}(z) \mathscr{L}_{\nabla, t_{0}}\left\{D_{\nabla}^{n} f(t)\right\}(z) \\
= & \frac{1}{z^{\alpha}}\left[z^{n} \mathscr{L}_{\nabla, t_{0}}\{f(t)\}(z)-\sum_{k=0}^{n-1} z^{n-k-1} f^{\nabla^{k}}\left(t_{0}\right)\right] \\
= & \frac{1}{z^{\alpha-n}} \mathscr{L}_{\nabla, t_{0}}\{f(t)\}(z)-\sum_{k=0}^{n-1} \frac{1}{z^{\alpha-n+k+1}} f^{\nabla^{k}}\left(t_{0}\right) \\
= & \mathscr{L}_{\nabla, t_{0}}\left\{\widehat{h}_{\alpha-n-1}\left(t, t_{0}\right) * f(t)\right\}(z) \\
& -\sum_{k=0}^{n-1} \mathscr{L}_{\nabla, t_{0}}\left\{\widehat{h}_{\alpha-n-k}\left(t, t_{0}\right)\right\}(z) f^{\nabla^{k}}\left(t_{0}\right) \\
= & \mathscr{L}_{\nabla, t_{0}}\left\{D_{\nabla, t_{0}}^{n} I^{\alpha}(t)-\sum_{k=0}^{n-1} \widehat{h}_{\alpha-n-k}\left(t, t_{0}\right) f^{\nabla^{k}}\left(t_{0}\right)\right\}(z) .
\end{aligned}
$$

Using the uniqueness of the inverse Laplace transform, we can derive that

$$
I_{\nabla, t_{0}}^{\alpha} D_{\nabla}^{n} f(t)=D_{\nabla}^{n} I_{\nabla, t_{0}}^{\alpha} f(t)-\sum_{k=0}^{n-1} \widehat{h}_{\alpha-n-k}\left(t, t_{0}\right) f^{\nabla^{k}}\left(t_{0}\right) .
$$

The proof is finished.

To present the next property, we use the space of function $I_{\nabla, t_{0}}^{\alpha}\left(L_{\nabla, p}\right)$ defined for $\alpha>0$ and $1 \leq p \leq \infty$ by

$$
I_{\nabla, t_{0}}^{\alpha}\left(L_{\nabla, p}\right):=\left\{f: f=I_{\nabla, t_{0}}^{\alpha} \varphi, \varphi \in L_{\nabla, p}(\Omega)\right\} .
$$

The composition of the fractional $\nabla$-integral operator $I_{\nabla, t_{0}}^{\alpha}$ with the fractional $\nabla$-differentiation operator $D_{\nabla, t_{0}}^{\alpha}$ is given by the following result.

Property 8. Let $\alpha>0, m=[\alpha]+1$ and let $f_{m-\alpha}(t)=I_{\nabla, t_{0}}^{m-\alpha} f(t)$.

(1) If $1 \leq p \leq \infty$ and $f(t) \in I_{\nabla, t_{0}}^{\alpha}\left(L_{\nabla, p}\right)$, then

$$
I_{\nabla, t_{0}}^{\alpha} D_{\nabla, t_{0}}^{\alpha} f(t)=f(t) \text {. }
$$

(2) If $f(t) \in L_{\nabla, 1}(\Omega)$ and $f_{m-\alpha}(t) \in A C_{\nabla}^{m}(\Omega)$, then the equality

$$
I_{\nabla, t_{0}}^{\alpha} D_{\nabla, t_{0}}^{\alpha} f(t)=f(t)-\sum_{k=1}^{m} \widehat{h}_{\alpha-k}\left(t, t_{0}\right) D_{\nabla, t_{0}}^{\alpha-k} f\left(t_{0}\right)
$$

holds almost everywhere on $\Omega$, where $D_{\nabla, t_{0}}^{\alpha-m} y\left(t_{0}\right)=$ $\lim _{t \rightarrow t_{0}^{+}} I_{\nabla, t_{0}}^{m-\alpha} y(t)$. 
Proof. (1) From the definition of $I_{\nabla, t_{0}}^{\alpha}\left(L_{\nabla, p}\right)$ and (101), it is easy to obtain the result. get

(2) Applying Laplace transform to $I_{\nabla, t_{0}}^{\alpha} D_{\nabla, t_{0}}^{\alpha} f(t)$, we can

$$
\begin{aligned}
\mathscr{L}_{\nabla, t_{0}}\left\{I_{\nabla, t_{0}}^{\alpha} D_{\nabla, t_{0}}^{\alpha} f(t)\right\}(z) \\
=\mathscr{L}_{\nabla, t_{0}}\left\{\widehat{h}_{\alpha-1}\left(t, t_{0}\right) * D_{\nabla, t_{0}}^{\alpha} f(t)\right\}(z) \\
=\mathscr{L}_{\nabla, t_{0}}\left\{\widehat{h}_{\alpha-1}\left(t, t_{0}\right)\right\}(z) \mathscr{L}_{\nabla, t_{0}}\left\{D_{\nabla, t_{0}}^{\alpha} f(t)\right\}(z) \\
=\frac{1}{z^{\alpha}}\left[z^{\alpha} \mathscr{L}_{\nabla, t_{0}}\{f(t)\}(z)-\sum_{k=1}^{m} z^{k-1} D_{\nabla, t_{0}}^{\alpha-k} f\left(t_{0}\right)\right] \\
=\mathscr{L}_{\nabla, t_{0}}\{f(t)\}(z)-\sum_{k=1}^{m} \frac{1}{z^{\alpha-k+1}} D_{\nabla, t_{0}}^{\alpha-k} f\left(t_{0}\right) \\
=\mathscr{L}_{\nabla, t_{0}}\{f(t)\}(z) \\
\quad-\sum_{k=1}^{m} \mathscr{L}_{\nabla, t_{0}}\left\{\widehat{h}_{\alpha-k}\left(t, t_{0}\right)\right\}(z) D_{\nabla, t_{0}}^{\alpha-k} f\left(t_{0}\right) \\
=\mathscr{L}_{\nabla, t_{0}}\left\{f(t)-\sum_{k=1}^{m} \widehat{h}_{\alpha-k}\left(t, t_{0}\right) D_{\nabla, t_{0}}^{\alpha-k} f\left(t_{0}\right)\right\}(z) .
\end{aligned}
$$

By the uniqueness of Laplace transform, we have

$$
I_{\nabla, t_{0}}^{\alpha} D_{\nabla, t_{0}}^{\alpha} f(t)=f(t)-\sum_{k=1}^{m} \widehat{h}_{\alpha-k}\left(t, t_{0}\right) D_{\nabla, t_{0}}^{\alpha-k} f\left(t_{0}\right)
$$

Property 9. Let $f_{M-\beta}(t)=I_{\nabla, t_{0}}^{M-\beta} f(t)$. When $\beta>\alpha>0, M=$ $[\beta]+1$, if $f(t) \in L_{\nabla, 1}(\Omega)$ and $f_{M-\beta}(t) \in A C^{M}(\Omega)$, then we have the following equation:

(1) $\quad D_{\nabla, t_{0}}^{\beta} I_{\nabla, t_{0}}^{\alpha} f(t)=D_{\nabla, t_{0}}^{\beta-\alpha} f(t)$,

(2) $\quad I_{\nabla, t_{0}}^{\alpha} D_{\nabla, t_{0}}^{\beta} f(t)=D_{\nabla, t_{0}}^{\beta-\alpha} f(t)$

$$
-\sum_{k=1}^{M} \widehat{h}_{\alpha-k}\left(t, t_{0}\right) D_{\nabla, t_{0}}^{\beta-k} f\left(t_{0}\right) \text {. }
$$

Proof. Let $m=[\alpha]+1$. According to Property 3 and the definition of fractional $\nabla$-derivative, we have

$$
\begin{aligned}
D_{\nabla, t_{0}}^{\beta} I_{\nabla, t_{0}}^{\alpha} f(t) & =D_{\nabla}^{M} I_{\nabla, t_{0}}^{M-\beta} I_{\nabla, t_{0}}^{\alpha} f(t) \\
& =D_{\nabla}^{M} I_{\nabla, t_{0}}^{M-\beta+\alpha} f(t) \\
& =D_{\nabla}^{M-m} D_{\nabla}^{m} I_{\nabla, t_{0}}^{m} I_{\nabla, t_{0}}^{(M-m)-(\beta-\alpha)} f(t) \\
& =D_{\nabla}^{M-m} I_{\nabla, t_{0}}^{(M-m)-(\beta-\alpha)} f(t) \\
& =D_{\nabla, t_{0}}^{\beta-\alpha} f(t) .
\end{aligned}
$$

In addition,

$$
\begin{aligned}
I_{\nabla, t_{0}}^{\alpha} D_{\nabla, t_{0}}^{\beta} f(t)= & I_{\nabla, t_{0}}^{\alpha} D_{\nabla}^{M} I_{\nabla, t_{0}}^{M-\beta} f(t) \\
= & D_{\nabla}^{M} I_{\nabla, t_{0}}^{\alpha} I_{\nabla, t_{0}}^{M-\beta} f(t) \\
& -\sum_{k=0}^{M-1} \widehat{h}_{\alpha-M+k}\left(t, t_{0}\right) D_{\nabla, t_{0}}^{k+\beta-M} f\left(t_{0}\right) \\
= & D_{\nabla, t_{0}}^{\beta-\alpha} f(t) \\
& -\sum_{k=1}^{M} \widehat{h}_{\alpha-k}\left(t, t_{0}\right) D_{\nabla, t_{0}}^{\beta-k} f\left(t_{0}\right) .
\end{aligned}
$$

Property 10. Let $f_{M-\beta}(t)=I_{\nabla, t_{0}}^{M-\beta} f(t)$ and $n-1<\alpha \leq n$, $m-1<\beta \leq m(n, m \in \mathbb{N})$. If $f(t) \in L_{\nabla, 1}(\Omega)$ and $f_{M-\beta}(t) \in$ $A C_{\nabla}^{M+m}(\Omega)$, then we have the following equation:

$$
D_{\nabla, t_{0}}^{\alpha} D_{\nabla, t_{0}}^{\beta} f(t)=D_{\nabla, t_{0}}^{\alpha+\beta} f(t)-\sum_{k=1}^{M} \widehat{h}_{-k-\alpha}\left(t, t_{0}\right) D_{\nabla, t_{0}}^{\beta-k} f\left(t_{0}\right) .
$$

Proof. According to Property 6 and Property 9, we have

$$
\begin{aligned}
D_{\nabla, t_{0}}^{\alpha} D_{\nabla, t_{0}}^{\beta} f(t)= & D_{\nabla, t_{0}}^{\alpha} D_{\nabla}^{M} I_{\nabla, t_{0}}^{M-\beta} f(t) \\
= & D_{\nabla, t_{0}}^{\alpha+M} I_{\nabla, t_{0}}^{M-\beta} f(t) \\
& -\sum_{k=0}^{M-1} \widehat{h}_{-\alpha-M+k}\left(t, t_{0}\right) D_{\nabla}^{k} I_{\nabla, t_{0}}^{M-\beta} f\left(t_{0}\right) \\
= & D_{\nabla, t_{0}}^{\alpha+\beta} f(t) \\
& -\sum_{k=0}^{M-1} \widehat{h}_{-\alpha-M+k}\left(t, t_{0}\right) D_{\nabla, t_{0}}^{k-M+\beta} f\left(t_{0}\right) \\
= & D_{\nabla, t_{0}}^{\alpha+\beta} f(t)-\sum_{k=1}^{M} \widehat{h}_{-k-\alpha}\left(t, t_{0}\right) D_{\nabla, t_{0}}^{\beta-k} f\left(t_{0}\right) .
\end{aligned}
$$

From Property 10, we derive the following result in [1] when $\mathbb{T}=\mathbb{R}$.

Corollary 45 (see [1]). Let $\alpha>0$ and $\beta>0$ be such that $n-1<$ $\alpha \leq n, m-1<\beta \leq m(n, m \in \mathbb{N})$ and $\alpha+\beta<n$, and let $f \in L_{1}(a, b)$ and $f_{m-\alpha} \in A C^{m}([a, b])$. Then, there is the following index rule:

$$
\begin{aligned}
\left(D_{a+}^{\alpha} D_{a+}^{\beta} f\right)(x)= & \left(D_{a+}^{\alpha+\beta} f\right)(x) \\
& -\sum_{j=1}^{m}\left(D_{a+}^{\beta-j} f\right)(a+) \frac{(x-a)^{-j-\alpha}}{\Gamma(1-j-\alpha)} .
\end{aligned}
$$


It follows from Property 10, for fractional sum and difference, that there is also the following theorem in [8].

Corollary 46 (see $[8])$. Let $p>0, M=[p]+1$. Then

$$
\begin{aligned}
n_{0} \nabla_{n}^{q}\left[n_{0} \nabla_{n}^{p} f(n)\right]={ }_{n_{0}} \nabla_{n}^{p+q} f(n) \\
\\
\quad-\sum_{k=1}^{M}\left[\begin{array}{c}
-q-k+1 \\
n-n_{0}
\end{array}\right]\left[{ }_{n_{0}} \nabla_{n}^{p-k} f\left(n_{0}-1\right)\right] .
\end{aligned}
$$

\section{Cauchy-Type Problem with \\ Riemann-Liouville Fractional Derivative}

In this section, we consider Cauchy-type problem with Riemann-Liouville fractional nabla derivative

$$
\begin{aligned}
& D_{\nabla, t_{0}}^{\alpha} y(t)=f(t, y(t)) \quad(\alpha>0), \\
& D_{\nabla, t_{0}}^{\alpha-k} y\left(t_{0}\right)=b_{k} \quad(k=1, \ldots, m),
\end{aligned}
$$

where $m=[\alpha]+1$ for $\alpha \notin \mathbb{N}$ and $\alpha=m$ for $\alpha \in \mathbb{N}$, and the notation $D_{\nabla, t_{0}}^{\alpha-k} y\left(t_{0}\right)$ means that if $t_{0}$ is right-dense, then

$$
\begin{aligned}
& D_{\nabla, t_{0}}^{\alpha-k} y\left(t_{0}\right)=\lim _{t \rightarrow t_{0}^{+}} D_{\nabla, t_{0}}^{\alpha-k} y(t) \quad(1 \leq k \leq m-1), \\
& D_{\nabla, t_{0}}^{\alpha-m} y\left(t_{0}\right)=\lim _{t \rightarrow t_{0}^{+}} I_{\nabla, t_{0}}^{m-\alpha} y(t) \quad(\alpha \neq m), \\
& D_{\nabla, t_{0}}^{0} y\left(t_{0}\right)=y\left(t_{0}\right) \quad(\alpha=m),
\end{aligned}
$$

if $t_{0}$ is right-scatter, then $D_{\nabla, t_{0}}^{\alpha-k} y\left(t_{0}\right)=0$.

We discuss this Cauchy-type problem in the space $L_{\nabla}^{\alpha}(\Omega)$ defined for $\alpha>0$ by

$$
L_{\nabla}^{\alpha}(\Omega):=\left\{y \in L_{\nabla}(\Omega): D_{\nabla, t_{0}}^{\alpha} \in L_{\nabla}(\Omega)\right\} .
$$

Here, $L_{\nabla}(\Omega):=L_{\nabla, 1}(\Omega)$ is the space of $\nabla$-Lebesgue summable functions in a finite interval $\Omega$.

In particular, if $\alpha=m \in \mathbb{N}$, then the problem in (126) and (127) is reduced to the usual Cauchy problem for the ordinary differential equation of order $m \in \mathbb{N}$ on the following time scales:

$$
\begin{gathered}
y^{\nabla^{m}}(t)=f(t, y(t)), \\
y^{\nabla^{m-k}}\left(t_{0}\right)=b_{k} \quad(k=1, \ldots, m) .
\end{gathered}
$$

In the following, we prove that Cauchy-type problem and the nonlinear Volterra integral equation are equivalent in the sense that, if $y(t) \in L_{\nabla}(\Omega)$ satisfies one of these relations, then it also satisfies the other.
Theorem 47. Let $\alpha>0, m=-[-\alpha], t_{0}, t \in \Omega_{k^{m}}$. Let $G$ be an open set in $\mathbb{R}$ and let $f: \Omega \times G \rightarrow \mathbb{R}$ be a function such that $f(t, y) \in L_{\nabla}(\Omega)$ for any $y \in G$. If $y(t) \in L_{\nabla}(\Omega)$, then Cauchytype problem (126) and (127) is equivalent of the following equation:

$$
\begin{aligned}
y(t)= & \sum_{k=1}^{m} \widehat{h}_{\alpha-k}\left(t, t_{0}\right) b_{k} \\
& +\int_{t_{0}}^{t} \widehat{h}_{\alpha-1}(t, \rho(\tau)) f(\tau, y(\tau)) \nabla \tau
\end{aligned}
$$

Proof. First we prove the necessity. Let $y(t) \in L_{\nabla}(\Omega)$ satisfy a.e. the relations (126) and (127). Since $f(t, y) \in L_{\nabla}(\Omega)$, (126) means that there exists a.e. on $\Omega$ the fractional nabla derivative $D_{\nabla, t_{0}}^{\alpha} y(t) \in L_{\nabla}(\Omega)$. According to

$$
\begin{gathered}
D_{\nabla, t_{0}}^{\alpha} y(t)=D_{\nabla}^{m} I_{\nabla, t_{0}}^{m-\alpha} y(t), \\
(m=-[-\alpha]), \quad I_{\nabla, t_{0}}^{0} y(t)=y(t),
\end{gathered}
$$

we have $I_{\nabla, t_{0}}^{m-\alpha} y(t) \in A C_{\nabla}^{m}(\Omega)$. Thus, we apply $I_{\nabla, t_{0}}^{\alpha}$ to both sides of (126) and, in accordance with (116), we have

$$
\begin{aligned}
I_{\nabla, t_{0}}^{\alpha} D_{\nabla, t_{0}}^{\alpha} y(t) & =y(t)-\sum_{k=1}^{m} \widehat{h}_{\alpha-k}\left(t, t_{0}\right) D_{\nabla, t_{0}}^{\alpha-k} y\left(t_{0}\right) \\
& =\int_{t_{0}}^{t} \widehat{h}_{\alpha-1}(t, \rho(\tau)) f(\tau, y(\tau)) \nabla \tau .
\end{aligned}
$$

Thus,

$$
y(t)=\sum_{k=1}^{m} \widehat{h}_{\alpha-k}\left(t, t_{0}\right) b_{k}+\int_{t_{0}}^{t} \widehat{h}_{\alpha-1}(t, \rho(\tau)) f(\tau, y(\tau)) \nabla \tau \text {. }
$$

Now, we prove the sufficiency. Let $y(t) \in L_{\nabla}(\Omega)$ satisfy a.e. (131). Applying the operator $D_{\nabla, t_{0}}^{\alpha}$ to both sides of (131), we have

$$
D_{\nabla, t_{0}}^{\alpha} y(t)=\sum_{k=1}^{m} D_{\nabla, t_{0}}^{\alpha} \widehat{h}_{\alpha-k}\left(t, t_{0}\right) b_{k}+D_{\nabla, t_{0}}^{\alpha} I_{\nabla, t_{0}}^{\alpha} f(t, y(t)) \text {. }
$$

From here, in accordance with the formula (85) and (101), we arrive at (131). 
Now, we show that the relations in (127) also hold. For this, applying the operators $D_{\nabla, t_{0}}^{\alpha-k}(k=1, \ldots, m)$ to both sides of (131) and using (83) and (103), we have

$$
\begin{aligned}
D_{\nabla, t_{0}}^{\alpha-k} y(t)= & \sum_{j=1}^{m} D_{\nabla, t_{0}}^{\alpha-k} \widehat{h}_{\alpha-j}\left(t, t_{0}\right) b_{j}+D_{\nabla, t_{0}}^{\alpha-k} I_{\nabla, t_{0}}^{\alpha} f(t, y(t)) \\
= & \sum_{j=1}^{k} D_{\nabla, t_{0}}^{\alpha-k} \widehat{h}_{\alpha-j}\left(t, t_{0}\right) b_{j} \\
& +\sum_{j=k+1}^{m} D_{\nabla, t_{0}}^{\alpha-k} \widehat{h}_{\alpha-j}\left(t, t_{0}\right) b_{j} \\
& +D_{\nabla, t_{0}}^{\alpha-k} I_{\nabla, t_{0}}^{\alpha} f(t, y(t)) \\
= & \sum_{j=1}^{k} \widehat{h}_{k-j}\left(t, t_{0}\right) b_{j}+0+I_{\nabla, t_{0}}^{k} f(t, y(t)) \\
= & \sum_{j=1}^{k} \widehat{h}_{k-j}\left(t, t_{0}\right) b_{j} \\
& +\int_{t_{0}}^{t} \widehat{h}_{k-1}(t, \rho(\tau)) f(\tau, y(\tau)) \nabla \tau .
\end{aligned}
$$

Thus, we obtain the relations in (127).

In the following, we establish the existence of a unique solution to the Cauchy-type problem (126)-(127) in the space $L_{\nabla}^{\alpha}(\Omega)$ defined in (129) under the conditions of Theorem 47, and an additional Lipschitzian-type condition on $f(t, y)$ with respect to the second variable, for all $t \in \Omega$ and for all $y_{1}, y_{2} \in$ $G \subset \mathbb{R}$,

$$
\left|f\left(t, y_{1}\right)-f\left(t, y_{2}\right)\right| \leq A\left|y_{1}-y_{2}\right| \quad(A>0),
$$

where $A>0$ does not depend on $t \in \Omega$. we will derive a unique solution to the Cauchy-problem (126)-(127).

Theorem 48. Let $\alpha>0, m=-[-\alpha]$. Let $G$ be an open set in $\mathbb{R}$ and let $f: \Omega \times G \rightarrow \mathbb{R}$ be a function such that $f(t, y) \in$ $L_{\nabla}(\Omega)$ for any $y \in G$. Let $f(t, y)$ satisfy the Lipschitzian condition (137) and $\max _{y \in G, t, s \in \Omega}\left\{|f(t, y)|,\left|\widehat{h}_{\alpha-1}(t, s)\right|\right\} \leq M$. Then, there exists a unique solution $y(t)$ to the Cauchy-type problem (126)-(127) in the space $L_{\nabla}^{\alpha}(\Omega)$.

Proof. Since the Cauchy-type problem (126)-(127) and the nonlinear Volterra integral equation (131) are equivalent, we only need to prove there exists a unique solution to (131).

We define function sequences as follows:

$$
\begin{aligned}
y_{l}(t)= & y_{0}(t) \\
& +\int_{t_{0}}^{t} \widehat{h}_{\alpha-1}(t, \rho(\tau)) f\left(\tau, y_{l-1}(\tau)\right) \nabla \tau \quad(l=1,2, \ldots),
\end{aligned}
$$

where

$$
y_{0}(t)=\sum_{k=1}^{m} \widehat{h}_{\alpha-k}\left(t, t_{0}\right) b_{k}
$$

We obtain by induction

$$
\left|y_{l}(t)-y_{l-1}(t)\right| \leq M^{l+1} A^{l-1} \widehat{h}_{l}\left(t, t_{0}\right) .
$$

In fact, for $l=1$, since $\max _{y \in G, t, s \in \Omega}\left\{|f(t, y)|,\left|\widehat{h}_{\alpha-1}(t, s)\right|\right\} \leq$ $M$, we have

$$
\begin{aligned}
\left|y_{1}(t)-y_{0}(t)\right| & \leq \int_{t_{0}}^{t}\left|\widehat{h}_{\alpha-1}(t, \rho(\tau))\right|\left|f\left(\tau, y_{l-1}(\tau)\right)\right| \nabla \tau \\
& \leq M^{2} \int_{t_{0}}^{t} \nabla \tau=M^{2} \widehat{h}_{1}\left(t, t_{0}\right) .
\end{aligned}
$$

If

$$
\left|y_{l-1}(t)-y_{l-2}(t)\right| \leq M^{l} A^{l-2} \widehat{h}_{l-1}\left(t, t_{0}\right) .
$$

Then,

$$
\begin{aligned}
\mid y_{l}(t) & -y_{l-1}(t) \mid \\
& \leq A \int_{t_{0}}^{t}\left|\widehat{h}_{\alpha-1}(t, \rho(\tau))\right|\left|y_{l-1}(\tau)-y_{l-2}(\tau)\right| \nabla \tau \\
& \leq A M \int_{t_{0}}^{t} M^{l} A^{l-2} \widehat{h}_{l-1}\left(\tau, t_{0}\right) \nabla \tau \\
& =M^{l+1} A^{l-1} \int_{t_{0}}^{t} \widehat{h}_{l-1}\left(\tau, t_{0}\right) \nabla \tau \\
& =M^{l+1} A^{l-1} \widehat{h}_{l}\left(t, t_{0}\right) .
\end{aligned}
$$

Thus, from Lemma 9, we have

$$
\begin{aligned}
& \sum_{j=1}^{\infty}\left|y_{j}(t)-y_{j-1}(t)\right| \\
& \leq \sum_{j=1}^{\infty} M^{j+1} A^{j-1} \widehat{h}_{j}\left(t, t_{0}\right) \\
& =\frac{M}{A} \sum_{j=1}^{\infty} M^{j} A^{j} \widehat{h}_{j}\left(t, t_{0}\right) \\
& =\frac{M}{A} \sum_{j=1}^{\infty} M^{j} A^{j} h_{j}\left(\sigma(t), t_{0}\right) \\
& \leq \frac{M}{A} \sum_{j=1}^{\infty} M^{j} A^{j} \frac{\left(\sigma(t)-t_{0}\right)^{j}}{j !} \\
& \leq \frac{M}{A} \sum_{j=1}^{\infty} M^{j} A^{j} \frac{\left(\sigma\left(t_{1}\right)-t_{0}\right)^{j}}{j !} .
\end{aligned}
$$


By Weierstrass discriminance, we obtain $y_{l}(t)$ convergent uniformly. Let $y(t)=\lim _{l \rightarrow \infty} y_{l}(t)$, then $y(t)$ is a solution of (131). Next, we will show the uniqueness. Assume that $z(t)$ is another solution to (131), that is,

$$
z(t)=y_{0}(t)+\int_{t_{0}}^{t} \widehat{h}_{\alpha-1}(t, \rho(\tau)) f(\tau, z(\tau)) \nabla \tau .
$$

As

$$
\begin{gathered}
\max _{y \in G, t, s \in \Omega}\left\{|f(t, y)|, \widehat{h}_{\alpha}(t, s)\right\} \leq M \\
\left|y_{0}(t)-z(t)\right| \leq \int_{t_{0}}^{t}\left|\widehat{h}_{\alpha-1}(t, \rho(\tau))\right||f(\tau, z(\tau))| \nabla \tau \\
\leq M^{2} \int_{t_{0}}^{t} \nabla \tau=M^{2} \widehat{h}_{1}\left(t, t_{0}\right) .
\end{gathered}
$$

If

$$
\left|y_{l-1}(t)-z(t)\right| \leq M^{l+1} A^{l-1} \widehat{h}_{l}\left(t, t_{0}\right),
$$

then

$$
\begin{aligned}
& \left|y_{l}(t)-z(t)\right| \\
& \quad \leq \int_{t_{0}}^{t}\left|\widehat{h}_{\alpha-1}(t, \rho(\tau))\right|\left|f\left(\tau, y_{l-1}(\tau)-f(\tau, z(\tau))\right)\right| \nabla \tau \\
& \quad \leq M A \int_{t_{0}}^{t}\left|y_{l-1}(\tau)-z(\tau)\right| \nabla \tau \\
& \quad \leq M A \int_{t_{0}}^{t} M^{l+1} A^{l-1} \widehat{h}_{l}\left(\tau, t_{0}\right) \nabla \tau \\
& \quad=M^{l+2} A^{l} \int_{t_{0}}^{t} \widehat{h}_{l}\left(\tau, t_{0}\right) \nabla \tau \\
& \quad=M^{l+2} A^{l} \widehat{h}_{l+1}\left(t, t_{0}\right) .
\end{aligned}
$$

By mathematical induction, we have

$$
\left|y_{l}(t)-z(t)\right| \leq M^{l+2} A^{l} \widehat{h}_{l+1}\left(t, t_{0}\right),
$$

and then, from Lemma 9, we get that

$$
\begin{aligned}
\sum_{l=0}^{\infty}\left|y_{l}(t)-z(t)\right| & \\
\leq & \frac{M}{A} \sum_{l=0}^{\infty}(M A)^{l+1} \widehat{h}_{l+1}\left(t, t_{0}\right) \\
= & \frac{M}{A} \sum_{l=0}^{\infty}(M A)^{l+1} h_{l+1}\left(\sigma(t), t_{0}\right) \\
\leq & \frac{M}{A} \sum_{l=0}^{\infty}(M A)^{l+1} \frac{\left(\sigma(t)-t_{0}\right)^{l+1}}{(l+1) !} .
\end{aligned}
$$

Thus, $\lim _{l \rightarrow \infty} y_{l}(t)=z(t)$, and then get $z(t)=y(t)$ owing to the uniqueness of the limit. To complete the proof of
Theorem 48 , we must show that such a unique solution $y(t) \epsilon$ $L_{\nabla}(\Omega)$ belongs to the space $L_{\nabla}^{\alpha}(\Omega)$. In accordance with (129), it is sufficient to prove that $D_{\nabla, t_{0}}^{\alpha} y(t) \in L_{\nabla}(\Omega)$. By the above proof, the solution $y(t) \in L_{\nabla}(\Omega)$ is a limit of the sequence $y_{m}(t) \in L_{\nabla}(\Omega)$ as follows

$$
\lim _{m \rightarrow \infty}\left\|y_{m}(t)-y(t)\right\|=0 .
$$

By (126) and (137), we have

$$
\begin{aligned}
\left\|D_{\nabla, t_{0}}^{\alpha} y_{m}(t)-D_{\nabla, t_{0}}^{\alpha} y(t)\right\| & =\left\|f\left(t, y_{m}\right)-f(t, y)\right\| \\
& \leq A\left\|y_{m}(t)-y(t)\right\| .
\end{aligned}
$$

Thus, by (151), we get

$$
\lim _{m \rightarrow \infty}\left\|D_{\nabla, t_{0}}^{\alpha} y_{m}(t)-D_{\nabla, t_{0}}^{\alpha} y(t)\right\|=0,
$$

and hence $D_{\nabla, t_{0}}^{\alpha} y(t) \in L_{\nabla}(\Omega)$. This completes the proof of Theorem 48.

Next, we consider the generalized Cauchy-type problem as follows:

$$
\begin{gathered}
D_{\nabla, t_{0}}^{\alpha} y(t)=f\left(t, y(t), D_{\nabla, t_{0}}^{\alpha_{1}} y(t), \ldots, D_{\nabla, t_{0}}^{\alpha_{l}} y(t)\right) \\
\left(0=\alpha_{0} \leq \alpha_{1} \leq \cdots \leq \alpha_{l}<\alpha\right), \\
D_{\nabla, t_{0}}^{\alpha-k} y\left(t_{0}\right)=b_{k}(k=1, \ldots, m, m=-[-\alpha]) .
\end{gathered}
$$

Theorem 49. Let $f: \Omega \times G \rightarrow \mathbb{R}$ be a function such that $f\left(t, y, y_{1}, \ldots, y_{l}\right) \in L_{\nabla}(\Omega)$ for any $\left(y, y_{1}, \ldots, y_{l}\right) \in G$. If $y(t) \in$ $L_{\nabla}(\Omega)$, then $y(t)$ satisfies a.e. the relations (154) if and only if satisfies a.e. the integral equation as follows

$$
\begin{aligned}
y(t)= & \sum_{k=1}^{m} \widehat{h}_{\alpha-k}\left(t, t_{0}\right) b_{k} \\
& +\int_{t_{0}}^{t} \widehat{h}_{\alpha-1}(t, \rho(\tau)) \\
& \times f\left(\tau, y(\tau), D_{\nabla, t_{0}}^{\alpha_{1}} y(\tau), \ldots, D_{\nabla, t_{0}}^{\alpha_{l}} y(\tau)\right) \nabla \tau .
\end{aligned}
$$

Assume that $f$ satisfies generalized Lipschitzian condition as follows:

$$
\begin{gathered}
\left|f\left(t, y, y_{1}, \ldots, y_{l}\right)-f\left(t, z, z_{1}, \ldots, z_{l}\right)\right| \\
\leq A\left[\sum_{j=1}^{l}\left|y_{j}-z_{j}\right|\right] \quad(A>0) .
\end{gathered}
$$

According to the theorem above and by a similar proof of Theorem 48, we have the following theorem

Theorem 50. Let the condition of Theorem 49 be valid and let $f\left(t, y, y_{1}, \ldots, y_{l}\right)$ satisfy the Lipschitzian condition (156) and $\max _{y \in G, t, s \in \Omega}\left\{\left|f\left(t, y, y_{1}, \ldots, y_{l}\right)\right| \widehat{h}_{\alpha}(t, s)\right\} \leq M$. Then there exists a unique solution $y(t)$ to be the generalized Cauchy-type problem in the space $L_{\nabla}^{\alpha}(\Omega)$. 


\section{The Dependency of the Solution upon the Initial Value}

We consider fractional differential initial value problem (126)-(127) again as follows

$$
\begin{gathered}
D_{\nabla, t_{0}}^{\alpha} y(t)=f(t, y(t)), \\
D_{\nabla, t_{0}}^{\alpha-k} y\left(t_{0}\right)=b_{k}(k=1, \ldots, m=-[-\alpha]),
\end{gathered}
$$

where $\alpha>0$.

Using Theorem 47, we have

$$
y(t)=y_{0}(t)+\int_{t_{0}}^{t} \widehat{h}_{\alpha-1}(t, \rho(\tau)) f(\tau, y(\tau)) \nabla \tau,
$$

where

$$
y_{0}(t)=\sum_{k=1}^{m} \widehat{h}_{\alpha-k}\left(t, t_{0}\right) b_{k}
$$

Suppose that $z(t)$ is the solution to the initial value problem as follows

$$
\begin{gathered}
D_{\nabla, t_{0}}^{\alpha} y(t)=f(t, y(t)), \\
D_{\nabla, t_{0}}^{\alpha-k} y\left(t_{0}\right)=c_{k} \quad(k=1, \ldots, m=-[-\alpha]) .
\end{gathered}
$$

We denote $\|y(t)\|:=\max _{t \in \Omega} y(t)$. We can derive the dependency of the solution upon the initial value.

Theorem 51. Let $t_{0}, t, s \in \Omega_{k}$, $\left|\widehat{h}_{\alpha-1}(t, s)\right| \leq M$ and suppose $f$ satisfy the Lipschitz condition, that is,

$$
|f(t, z)-f(t, y)| \leq A|z-y| \quad(A>0) .
$$

Then, we have

$$
|z(t)-y(t)| \leq\left\|z_{0}-y_{0}\right\| \sum_{j=0}^{\infty} M^{j} A^{j} \frac{\left(\rho(t)-t_{0}\right)^{j}}{j !} .
$$

Proof. By the proof of Theorem 48, we know that $y(t)=$ $\lim _{m \rightarrow \infty} y_{m}(t), z(t)=\lim _{m \rightarrow \infty} z_{m}(t)$, where

$$
\begin{aligned}
& y_{0}(t)=\sum_{k=1}^{m} \widehat{h}_{\alpha-k}\left(t, t_{0}\right) b_{k}, \\
& y_{m}(t)=y_{0}(t)+\int_{t_{0}}^{t} \widehat{h}_{\alpha-1}(t, \rho(\tau)) f\left(\tau, y_{m-1}(\tau)\right) \nabla \tau, \\
& z_{0}(t)=\sum_{k=1}^{m} \widehat{h}_{\alpha-k}\left(t, t_{0}\right) c_{k}, \\
& z_{m}(t)=z_{0}(t)+\int_{t_{0}}^{t} \widehat{h}_{\alpha-1}(t, \rho(\tau)) f\left(\tau, z_{m-1}(\tau)\right) \nabla \tau .
\end{aligned}
$$

Using the Lipschitz condition, we have

$$
\begin{aligned}
\mid z_{1}(t) & -y_{1}(t) \mid \\
\leq & \left\|z_{0}-y_{0}\right\| \\
& +\int_{t_{0}}^{t}\left|\widehat{h}_{\alpha-1}(t, \rho(\tau))\right|\left|f\left(\tau, z_{0}(\tau)\right)-f\left(\tau, y_{0}(\tau)\right)\right| \nabla \tau \\
\leq & \left\|z_{0}-y_{0}\right\|+M \int_{t_{0}}^{t} A\left\|z_{0}-y_{0}\right\| \nabla \tau \\
= & \left\|z_{0}-y_{0}\right\|+M A\left\|z_{0}-y_{0}\right\| \int_{t_{0}}^{t} \nabla \tau \\
= & \left\|z_{0}-y_{0}\right\|+M A\left\|z_{0}-y_{0}\right\| \widehat{h}_{1}\left(t, t_{0}\right) \\
= & \left\|z_{0}-y_{0}\right\|\left(1+M A \widehat{h}_{1}\left(t, t_{0}\right)\right) .
\end{aligned}
$$

Suppose that

$$
\begin{aligned}
& \left|z_{m-1}(t)-y_{m-1}(t)\right| \\
& \quad \leq\left\|z_{0}-y_{0}\right\| \sum_{j=0}^{m-1}(M A)^{j} \widehat{h}_{j}\left(t, t_{0}\right) .
\end{aligned}
$$

Then,

$$
\begin{aligned}
\left|z_{m}(t)-y_{m}(t)\right| & \\
\leq & \left\|z_{0}-y_{0}\right\| \\
& +\int_{t_{0}}^{t}\left|\widehat{h}_{\alpha-1}(t, \rho(\tau))\right|\left|f\left(\tau, z_{m-1}(\tau)\right)-f\left(\tau, y_{m-1}(\tau)\right)\right| \nabla \tau \\
\leq & \left\|z_{0}-y_{0}\right\| \\
& +M \int_{t_{0}}^{t} A\left|z_{m-1}(\tau)-y_{m-1}(\tau)\right| \nabla \tau \\
\leq & \left\|z_{0}-y_{0}\right\| \\
& +M \int_{t_{0}}^{t} A\left\|z_{0}-y_{0}\right\| \sum_{j=0}^{m-1}(M A)^{j} \widehat{h}_{j}\left(\tau, t_{0}\right) \nabla \tau \\
= & \left\|z_{0}-y_{0}\right\| \\
& +M A\left\|z_{0}-y_{0}\right\| \sum_{j=0}^{m-1}(M A)^{j} \int_{t_{0}}^{t} \widehat{h}_{j}\left(\tau, t_{0}\right) \nabla \tau \\
= & \left\|z_{0}-y_{0}\right\| \\
& +M A\left\|z_{0}-y_{0}\right\| \sum_{j=0}^{m-1}(M A)^{j} \widehat{h}_{j+1}\left(t, t_{0}\right) \\
= & \left\|z_{0}-y_{0}\right\| \sum_{j=0}^{m}(M A)^{j} \widehat{h}_{j}\left(t, t_{0}\right) .
\end{aligned}
$$


According to mathematical induction, we have

$$
\left|z_{m}(t)-y_{m}(t)\right| \leq\left\|z_{0}-y_{0}\right\| \sum_{j=0}^{m}(M A)^{j} \widehat{h}_{j}\left(t, t_{0}\right) .
$$

Taking the limit $m \rightarrow \infty$ and from Lemma 9 , we obtain that

$$
\begin{aligned}
|z(t)-y(t)| & \leq\left\|z_{0}-y_{0}\right\| \sum_{j=0}^{\infty}(M A)^{j} \widehat{h}_{j}\left(t, t_{0}\right) \\
& =\left\|z_{0}-y_{0}\right\| \sum_{j=0}^{\infty}(M A)^{j} h_{j}\left(\sigma(t), t_{0}\right) \\
& \leq\left\|z_{0}-y_{0}\right\| \sum_{j=0}^{\infty}(M A)^{j} \frac{\left(\sigma(t)-t_{0}\right)^{j}}{j !} .
\end{aligned}
$$

As a special case, when fractional equation is linear, we can obtain its explicit solutions and we will explain it in the next section.

\section{Homogeneous Equations with Constant Coefficients}

In this section, we apply the Laplace transform method to derive the fundamental system of solutions to homogeneous equations of the following form:

$$
\begin{gathered}
\sum_{k=1}^{m} A_{k}\left[D_{\nabla, t_{0}}^{\alpha_{k}} y(t)\right]+A_{0} y(t)=0 \\
\left(t>t_{0} ; m \in \mathbb{N} ; 0<\alpha_{1}<\cdots<\alpha_{m}\right),
\end{gathered}
$$

with the Riemann-Liouville fractional derivatives $D_{\nabla, t_{0}}^{\alpha_{k}} y(k=$ $1, \ldots, m)$. Here, $A_{k} \in \mathbb{R}(k=0, \ldots, m)$ are real constants, and, generally speaking, we can take $A_{m}=1$.

The Laplace transform method is based on the relation (75) which is equivalent to the following one:

$$
\begin{gathered}
\mathscr{L}_{\nabla, t_{0}}\left\{D_{\nabla, t_{0}}^{\alpha} f(t)\right\}(z)=z^{\alpha} \mathscr{L}_{\nabla, t_{0}}\{f(t)\}(z) \\
-\sum_{j=1}^{l} d_{j} z^{j-1}(l-1<\alpha \leq l ; l \in \mathbb{N}), \\
d_{j}=D_{\nabla, t_{0}}^{\alpha-j} f\left(t_{0}\right) \quad(j=1, \ldots, l) .
\end{gathered}
$$

First, we derive explicit solutions to (169) with $m=1$ as follows:

$$
\begin{gathered}
D_{\nabla, t_{0}}^{\alpha} y(t)-\lambda y(t)=0 \\
\left(t>t_{0} ; l-1<\alpha \leq l ; l \in \mathbb{N} ; \lambda \in \mathbb{R}\right) .
\end{gathered}
$$

In order to prove our result, we also need the following definition and lemma.

Definition 52. The function $W_{\alpha}(t)$ is defined by

$$
W_{\alpha}(t)=\operatorname{det}\left(\left(D_{\nabla, t_{0}}^{\alpha-k} y_{j}\right)(t)\right)_{k, j=1}^{n} \quad(n=[\alpha]+1, t \in \Omega) .
$$

Lemma 53. The solutions $y_{1}(t), y_{2}(t), \ldots, y_{n}(t)$ are linearly independent if and only if $W_{\alpha}\left(t^{*}\right) \neq 0$ at some point $t^{*} \in \Omega$.

Proof. We first prove sufficiency. If, to the contrary, $y_{j}(t)(j=$ $1,2, \ldots, n)$ are linearly dependent in $\Omega$, then there exists $n$ constants $\left\{c_{j}\right\}_{j=1}^{n}$, not all zero, such that,

$$
\left(\left(D_{\nabla, t_{0}}^{\alpha-k} y_{j}\right)(t)\right)_{k, j=1}^{n}\left(\begin{array}{c}
c_{1} \\
c_{2} \\
\cdots \\
c_{n}
\end{array}\right) \equiv 0
$$

holds, and thus, $W_{\alpha}(t) \equiv 0$ which leads to a contradiction. Therefore, if $W_{\alpha}\left(t^{*}\right) \neq 0$ at some point $t^{*} \in \Omega$, then $y_{1}(t), y_{2}(t), \ldots, y_{n}(t)$ are linearly independent. Now, we prove necessity. Suppose, to the contrary, for $t \in \Omega, W_{\alpha}(t)=0$. Consider the following equations:

$$
\left(\left(D_{\nabla, t_{0}}^{\alpha-k} y_{j}\right)\left(t^{*}\right)\right)_{k, j=1}^{n} C=0
$$

where $t^{*} \in \Omega, C=\left(\begin{array}{c}c_{1} \\ c_{2} \\ \ldots \\ c_{n}\end{array}\right)$. As $W_{\alpha}\left(t^{*}\right)=0$, the equations has nontrivial solution $c_{j}(j=1,2, \ldots, n)$. Now we construct a function using these constants:

$$
y(t)=\sum_{j=1}^{n} c_{j} y_{j}(t)
$$

and we get that $y(t)$ is a solution. From (175), we obtain that $y(t)$ satisfies the following initial value condition:

$$
\left(D_{\nabla, t_{0}}^{\alpha-k} y\left(t^{*}\right)\right)=0, \quad k=1, \ldots, n .
$$

However, $y(t)=0$ is also a solution to equation satisfying the initial value condition. By the uniqueness of solution, we have

$$
\sum_{j=1}^{n} c_{j} y_{j}(t)=0
$$

and thus, $y_{j}(t)(j=1,2, \ldots, n)$ are linearly dependant which leads to a contradiction. Thus, if the solutions $y_{1}(t), y_{2}(t), \ldots$, $y_{n}(t)$ are linearly independent, then $W_{\alpha}\left(t^{*}\right) \neq 0$ at some point $t^{*} \in \Omega$.

There holds the following statements.

Theorem 54. Let $l-1<\alpha \leq l(l \in \mathbb{N})$ and $\lambda \in \mathbb{R}$. Then, the following functions:

$$
\begin{aligned}
y_{j}(t) & ={ }_{\nabla} F_{\alpha, \alpha+1-j}\left(\lambda ; t, t_{0}\right) \\
& =\sum_{k=0}^{\infty} \lambda^{k} \widehat{h}_{k \alpha+\alpha-j}\left(t, t_{0}\right) \quad(j=1, \ldots, l)
\end{aligned}
$$


yield the fundamental system of solutions to (172). Moreover, $y_{j}(t), j=1,2, \ldots, l$ satisfy

$$
\begin{gathered}
D_{\nabla, t_{0}}^{\alpha-k} y_{j}\left(t_{0}\right)=0 \quad(k, j=1, \ldots, l ; k \neq j), \\
D_{\nabla, t_{0}}^{\alpha-k} y_{k}\left(t_{0}\right)=1 \quad(k=1, \ldots, l) .
\end{gathered}
$$

Proof. Applying the Laplace transform to (172) and taking (170) into account, we have

$$
\mathscr{L}_{\nabla, t_{0}}\{y(t)\}(z)=\sum_{j=1}^{l} d_{j} \frac{z^{j-1}}{z^{\alpha}-\lambda},
$$

where $d_{j}(j=1, \ldots, l)$ are given by $(171)$.

Formula (79) with $\beta=\alpha+1-j$ yields

$$
\mathscr{L}_{\nabla, t_{0}}\left\{{ }_{\nabla} F_{\alpha, \alpha+1-j}\left(\lambda ; t, t_{0}\right)\right\}(z)=\frac{z^{j-1}}{z^{\alpha}-\lambda}\left(|\lambda|<|z|^{\alpha}\right) .
$$

Thus, from (181), we derive the following solution to (172) as follows:

$$
y(t)=\sum_{j=1}^{l} d_{j} y_{j}(t), y_{j}(t)={ }_{\nabla} F_{\alpha, \alpha+1-j}\left(\lambda ; t, t_{0}\right),
$$

which shows that an arbitrary solution $y(t)$ can be represented by $y_{j}(t), j=1, \ldots, l$. It is easily verified that the functions $y_{j}(t)$ are solutions to (172) as follows:

$$
\begin{aligned}
D_{\nabla, t_{0}}^{\alpha} & {\left[{ }_{\nabla} F_{\alpha, \alpha+1-j}\left(\lambda ; t, t_{0}\right)\right] } \\
& =\lambda_{\nabla} F_{\alpha, \alpha+1-j}\left(\lambda ; t, t_{0}\right) \quad(j=1, \ldots, l) .
\end{aligned}
$$

In fact,

$$
\begin{aligned}
D_{\nabla, t_{0}}^{\alpha}\left[{ }_{\nabla} F_{\alpha, \alpha+1-j}\left(\lambda ; t, t_{0}\right)\right] \\
=D_{\nabla, t_{0}}^{\alpha}\left[\sum_{k=0}^{\infty} \lambda^{k} \widehat{h}_{k \alpha+\alpha-j}\left(t, t_{0}\right)\right] \\
=D_{\nabla, t_{0}}^{\alpha}\left[\widehat{h}_{\alpha-j}\left(t, t_{0}\right)+\sum_{k=1}^{\infty} \lambda^{k} \widehat{h}_{k \alpha+\alpha-j}\left(t, t_{0}\right)\right] \\
\left.=D_{\nabla, t_{0}}^{\alpha} \widehat{h}_{\alpha-j}\left(t, t_{0}\right)+\sum_{k=1}^{\infty} \lambda^{k} \widehat{h}_{k \alpha-j}\left(t, t_{0}\right)\right] \\
=0+\sum_{k=0}^{\infty} \lambda^{k+1} \widehat{h}_{(k+1) \alpha-j}\left(t, t_{0}\right) \\
=\lambda \sum_{k=0}^{\infty} \lambda^{k} \widehat{h}_{k \alpha+\alpha-j}\left(t, t_{0}\right) \\
=\lambda_{\nabla} F_{\alpha, \alpha+1-j}\left(\lambda ; t, t_{0}\right) .
\end{aligned}
$$

Moreover,

$$
\begin{aligned}
D_{\nabla, t_{0}}^{\alpha-k} y_{j}(t) & =D_{\nabla, t_{0}}^{\alpha-k}\left[\sum_{s=0}^{\infty} \lambda^{s} \widehat{h}_{s \alpha+\alpha-j}\left(t, t_{0}\right)\right] \\
& =\sum_{s=0}^{\infty} \lambda^{s} \widehat{h}_{s \alpha+k-j}\left(t, t_{0}\right) .
\end{aligned}
$$

It follows from (186) that

$$
\begin{gathered}
D_{\nabla, t_{0}}^{\alpha-k} y_{j}\left(t_{0}\right)=0 \quad(k, j=1, \ldots, l ; k>j), \\
D_{\nabla, t_{0}}^{\alpha-k} y_{k}\left(t_{0}\right)=1 \quad(k=1, \ldots, l) .
\end{gathered}
$$

If $k<j$, then

$$
\begin{aligned}
D_{\nabla, t_{0}}^{\alpha-k} y_{j}(t) & \\
= & D_{\nabla, t_{0}}^{\alpha-k} \widehat{h}_{\alpha-j}\left(t, t_{0}\right)+\sum_{s=1}^{\infty} \lambda^{s} \widehat{h}_{s \alpha+k-j}\left(t, t_{0}\right) \\
= & D_{\nabla, t_{0}}^{m-k} I_{\nabla, t_{0}}^{m-\alpha} \widehat{h}_{\alpha-j}\left(t, t_{0}\right)+\sum_{s=1}^{\infty} \lambda^{s} \widehat{h}_{s \alpha+k-j}\left(t, t_{0}\right) \\
= & D_{\nabla, t_{0}}^{m-k} \widehat{h}_{m-j}\left(t, t_{0}\right)+\sum_{s=0}^{\infty} \lambda^{s+1} \widehat{h}_{s \alpha+\alpha+k-j}\left(t, t_{0}\right) \\
= & \sum_{s=0}^{\infty} \lambda^{s+1} \widehat{h}_{s \alpha+\alpha+k-j}\left(t, t_{0}\right),
\end{aligned}
$$

and since $\alpha+k-j \geq \alpha+1-l>0$ for any $k, j=1, \ldots, l$, the following relations hold:

$$
D_{\nabla, t_{0}}^{\alpha-k} y_{j}\left(t_{0}\right)=0 \quad(k, j=1, \ldots, l ; k<j) .
$$

By (187) and (189), $W_{\alpha}\left(t_{0}\right)=1$. Then, $y_{j}(t), j=1, \ldots, l$ which are linearly independent yield the fundamental system of solutions to (172).

Corollary 55. The following equation:

$$
D_{\nabla, t_{0}}^{\alpha} y(t)-\lambda y(t)=0 \quad\left(t>t_{0} ; 0<\alpha \leq 1 ; \lambda \in \mathbb{R}\right)
$$

has its solution given by

$$
y(t)={ }_{\nabla} F_{\alpha, \alpha}\left(\lambda ; t, t_{0}\right)
$$

while the equation

$$
D_{\nabla, t_{0}}^{\alpha} y(t)-\lambda y(t)=0 \quad\left(t>t_{0} ; 1<\alpha \leq 2 ; \lambda \in \mathbb{R}\right)
$$

has the fundamental system of solutions given by

$$
y_{1}(t)={ }_{\nabla} F_{\alpha, \alpha}\left(\lambda ; t, t_{0}\right), \quad y_{2}(t)={ }_{\nabla} F_{\alpha, \alpha-1}\left(\lambda ; t, t_{0}\right) .
$$

Next, we derive the explicit solutions to (169) with $m=2$ of the following form:

$$
\begin{gathered}
D_{\nabla, t_{0}}^{\alpha} y(t)-\lambda D_{\nabla, t_{0}}^{\beta} y(t)-\mu y(t)=0 \\
\left(t>t_{0} ; l-1<\alpha \leq l ; l \in \mathbb{N} ; 0<\beta<\alpha\right),
\end{gathered}
$$

with $\lambda, \mu \in \mathbb{R}$. 
Theorem 56. Let $l-1<\alpha \leq l(l \in \mathbb{N}), 0<\beta<\alpha$ and $\lambda, \mu \in \mathbb{R}$. Then, the following functions:

$$
\begin{array}{r}
y_{j}(t)=\sum_{k=0}^{\infty} \frac{\mu^{k}}{k !}\left\{\frac{\partial^{k}}{\partial \lambda^{k}} \nabla F_{\alpha-\beta, \alpha+k \beta+1-j}\left(\lambda ; t, t_{0}\right)\right\} \\
(j=1, \ldots, l)
\end{array}
$$

yield the fundamental system of solutions to (194), provided that the series in (195) is convergent. Moreover, if $\alpha+1-l>$ $\beta>l-1$, then, $y_{j}(t), j=1,2, \ldots, l$, in (195) satisfy (180).

Proof. Let $m-1<\beta \leq m(m \leq l ; m \in \mathbb{N})$. Applying the Laplace transform to (194) and using (170) as in (181), we obtain

$$
\mathscr{L}_{\nabla, t_{0}}\{y(t)\}(z)=\sum_{j=1}^{l} d_{j} \frac{z^{j-1}}{z^{\alpha}-\lambda z^{\beta}-\mu},
$$

where $d_{j}=D_{\nabla, t_{0}}^{\alpha-j} y\left(t_{0}\right)-\lambda D_{\nabla, t_{0}}^{\beta-j} y\left(t_{0}\right)(j=1, \ldots, m), d_{j}=$ $D_{\nabla, t_{0}}^{\alpha-j} y\left(t_{0}\right)(j=m+1, \ldots, l)$.

For $z \in \mathbb{C}$ and $\left|\mu z^{-\beta} /\left(z^{\alpha-\beta}-\lambda\right)\right|<1$, we have

$$
\begin{aligned}
\frac{1}{z^{\alpha}-\lambda z^{\beta}-\mu} & =\frac{z^{-\beta}}{z^{\alpha-\beta}-\lambda} \cdot \frac{1}{1-\left(\mu z^{-\beta} /\left(z^{\alpha-\beta}-\lambda\right)\right)} \\
& =\sum_{k=0}^{\infty} \frac{\mu^{k} z^{-\beta-k \beta}}{\left(z^{\alpha-\beta}-\lambda\right)^{k+1}},
\end{aligned}
$$

and hence (196) has the following representation:

$$
\mathscr{L}_{\nabla, t_{0}}\{y(t)\}(z)=\sum_{j=1}^{l} d_{j} \sum_{k=0}^{\infty} \mu^{k} \frac{z^{j-1-\beta-k \beta}}{\left(z^{\alpha-\beta}-\lambda\right)^{k+1}} .
$$

By (81), for $z \in \mathbb{C}$ and $\left|\lambda z^{\beta-\alpha}\right|<1$, we have

$$
\begin{aligned}
& \frac{z^{j-1-\beta-k \beta}}{\left(z^{\alpha-\beta}-\lambda\right)^{k+1}} \\
& \quad=\frac{z^{(\alpha-\beta)-(\alpha+k \beta+1-j)}}{\left(z^{\alpha-\beta}-\lambda\right)^{k+1}} \\
& =\frac{1}{k !} \mathscr{L}_{\nabla, t_{0}}\left\{\frac{\partial^{k}}{\partial \lambda^{k} \nabla} F_{\alpha-\beta, \alpha+k \beta+1-j}\left(\lambda ; t, t_{0}\right)\right\}(z) .
\end{aligned}
$$

From (198) and (199), we derive the solution to (194) as follows

$$
y(t)=\sum_{j=1}^{l} d_{j} y_{j}(t)
$$

which shows that an arbitrary solution $y(t)$ can be represented by $y_{j}(t), j=1, \ldots, l$, where $y_{j}(t)(j=1, \ldots, l)$ are given by (195). For $q, j=1, \ldots, l$, the direct evaluation yields

$$
\begin{aligned}
D_{\nabla, t_{0}}^{\alpha-q} y_{j}(t) & \\
= & D_{\nabla, t_{0}}^{\alpha-q} \sum_{k=0}^{\infty} \frac{\mu^{k}}{k !} \frac{\partial^{k}}{\partial \lambda^{k}}{ }_{\nabla} F_{\alpha-\beta, \alpha+k \beta+1-j}\left(\lambda ; t, t_{0}\right) \\
= & \sum_{k=0}^{\infty} \frac{\mu^{k}}{k !} \frac{\partial^{k}}{\partial \lambda^{k}} D_{\nabla, t_{0}}^{\alpha-q}\left[\sum_{s=0}^{\infty} \lambda^{s} \widehat{h}_{s(\alpha-\beta)+\alpha+k \beta-j}\left(t, t_{0}\right)\right] \\
= & \sum_{k=0}^{\infty} \frac{\mu^{k}}{k !} \frac{\partial^{k}}{\partial \lambda^{k}} \sum_{s=0}^{\infty} \lambda^{s} \widehat{h}_{s(\alpha-\beta)+k \beta+q-j}\left(t, t_{0}\right) .
\end{aligned}
$$

For $q>j, D_{\nabla, t_{0}}^{\alpha-q} y_{j}\left(t_{0}\right)=0$, and for $q=j, D_{\nabla, t_{0}}^{\alpha-q} y_{j}\left(t_{0}\right)=1$. Thus, we have $W_{\alpha}\left(t_{0}\right)=1$. It follows from Lemma 53 that the functions $y_{j}(t), j=1,2, \ldots, l$ in (195) are linearly independent solutions, and then they yield the fundamental system of solutions to (194). Furthermore, if $q<j$, then we rewrite (201) as follows:

$$
\begin{aligned}
D_{\nabla, t_{0}}^{\alpha-q} y_{j}(t)= & D_{\nabla, t_{0}}^{\alpha-q} \widehat{h}_{\alpha-j}\left(t, t_{0}\right) \\
& +\sum_{s=1}^{\infty} \lambda^{s} \widehat{h}_{s(\alpha-\beta)+q-j}\left(t, t_{0}\right) \\
& +\sum_{k=1}^{\infty} \frac{\mu^{k}}{k !} \frac{\partial^{k}}{\partial \lambda^{k}} \widehat{h}_{k \beta+q-j}\left(t, t_{0}\right) \\
& +\sum_{k=1}^{\infty} \frac{\mu^{k}}{k !} \frac{\partial^{k}}{\partial \lambda^{k}} \sum_{s=1}^{\infty} \lambda^{s} \widehat{h}_{s(\alpha-\beta)+k \beta+q-j}\left(t, t_{0}\right) \\
= & \sum_{s=1}^{\infty} \lambda^{s} \widehat{h}_{s(\alpha-\beta)+q-j}\left(t, t_{0}\right) \\
& +\sum_{k=1}^{\infty} \frac{\mu^{k}}{k !} \frac{\partial^{k}}{\partial \lambda^{k}} \widehat{h}_{k \beta+q-j}\left(t, t_{0}\right) \\
& +\sum_{k=1}^{\infty} \frac{\mu^{k}}{k !} \frac{\partial^{k}}{\partial \lambda^{k}} \sum_{s=1}^{\infty} \lambda^{s} \widehat{h}_{s(\alpha-\beta)+k \beta+q-j}\left(t, t_{0}\right) .
\end{aligned}
$$

If $\alpha+1-l>\beta>l-1$, then $s(\alpha-\beta)+q-j \geq(\alpha-\beta)+1-l>0$ for $k=0, q, j=1, \ldots, l, s \in \mathbb{N}^{+}$, and $k \beta+q-j \geq \beta+1-l>$ 0 for $s=0, q, j=1, \ldots, l, k \in \mathbb{N}^{+}$. Besides, we also have $s(\alpha-\beta)+k \beta+q-j \geq(\alpha-\beta)+\beta+1-l=\alpha+1-l>0$ for $q, j=1, \ldots, l, s, k \in \mathbb{N}^{+}$. These imply that $D_{\nabla, t_{0}}^{\alpha-q} y_{j}\left(t_{0}\right)=0$. Thus, the relations in (180) are valid. The proof is finished.

Corollary 57. The following equation form:

$$
\begin{gathered}
D_{\nabla, t_{0}}^{\alpha} y(t)-\lambda D_{\nabla, t_{0}}^{\beta} y(t)=0 \\
\left(t>t_{0} ; l-1<\alpha \leq l ; l \in \mathbb{N} ; 0<\beta<\alpha\right)
\end{gathered}
$$

has its fundamental system of solution given by

$$
y_{j}(t)={ }_{\nabla} F_{\alpha-\beta, \alpha+1-j}\left(\lambda ; t, t_{0}\right) \quad(j=1, \ldots, l) .
$$


Finally, we find the fundamental system of solutions to (169) with any $m \in \mathbb{N} \backslash\{1,2\}$. It is convenient to rewrite (169) in the form

$$
\begin{gathered}
D_{\nabla, t_{0}}^{\alpha} y(t)-\lambda D_{\nabla, t_{0}}^{\beta} y(t)-\sum_{k=0}^{m-2} A_{k} D_{\nabla, t_{0}}^{\alpha_{k}} y(t)=0 \\
\left(t>t_{0} ; m \in \mathbb{N} \backslash\{1,2\} ;\right. \\
0=\alpha_{0}<\alpha_{1}<\cdots<\alpha_{m-2}<\beta<\alpha ; \\
\left.\lambda, A_{0}, \ldots, A_{m-2} \in \mathbb{R}\right) .
\end{gathered}
$$

Theorem 58. Let $m \in \mathbb{N} \backslash\{1,2\}, l-1<\alpha \leq l(l \in \mathbb{N})$ and let $\beta$ and $\alpha_{1}, \ldots, \alpha_{m-2}$ be such that $\alpha>\beta>\alpha_{m-2}>\cdots>\alpha_{1}>\alpha_{0}=$ 0 , and let $\lambda, A_{0}, \ldots, A_{m-2} \in \mathbb{R}$. Then, the following functions:

$y_{j}(t)$

$$
\begin{aligned}
= & \sum_{n=0}^{\infty}\left(\sum_{k_{0}+\cdots+k_{m-2}=n}\right) \frac{1}{k_{0} ! \cdots k_{m-2} !}\left[\prod_{\nu=0}^{m-2}\left(A_{\nu}\right)^{k_{\nu}}\right] \\
& \cdot \frac{\partial^{n}}{\partial \lambda^{n}} F_{\alpha-\beta, \alpha+1-j+\sum_{\nu=0}^{m-2}\left(\beta-\alpha_{\nu}\right) k_{\nu}}\left(\lambda, t, t_{0}\right) \quad(j=1, \ldots, l)
\end{aligned}
$$

yield the fundamental system of solutions to (205), provided that the series in (206) are convergent. The inner sum is taken over all $k_{0}, \ldots, k_{m-2} \in \mathbb{N}_{0}$ such that $k_{0}+\cdots+k_{m-2}=n$. Moreover, if $\alpha+1-l>\beta>\alpha_{m-2}+l-1$, then $y_{j}(t), j=1,2, \ldots, l$, in (206) satisfy (180).

Proof. Let $l_{m-1}-1<\beta \leq l_{m-1}, l_{k}-1<\alpha_{k} \leq l_{k}(k=1, \ldots, m-2$; $\left.0 \leq l_{1} \leq \cdots \leq l_{m-1} \leq l\right)$. Applying the Laplace transform to (205) and using (170) as in (196), we obtain

$$
\mathscr{L}_{\nabla, t_{0}}\{y(t)\}(z)=\sum_{j=1}^{l} d_{j} \frac{z^{j-1}}{z^{\alpha}-\lambda z^{\beta}-\sum_{k=0}^{m-2} A_{k} z^{\alpha_{k}}},
$$

where

$$
\begin{aligned}
d_{j}= & D_{\nabla, t_{0}}^{\alpha-j} y\left(t_{0}\right)-\lambda D_{\nabla, t_{0}}^{\beta-j} y\left(t_{0}\right) \\
& -\sum_{k=1}^{m-2} A_{k} D_{\nabla, t_{0}}^{\alpha_{k}-j} y\left(t_{0}\right) \quad\left(j=1, \ldots, l_{1}\right), \\
d_{j}= & D_{\nabla, t_{0}}^{\alpha-j} y\left(t_{0}\right)-\lambda D_{\nabla, t_{0}}^{\beta-j} y\left(t_{0}\right) \\
& -\sum_{k=2}^{m-2} A_{k} D_{\nabla, t_{0}}^{\alpha_{k}-j} y\left(t_{0}\right) \quad\left(j=l_{1}+1, \ldots, l_{2}\right), \\
& \ldots \quad \quad d_{j}=D_{\nabla, t_{0}}^{\alpha-j} y\left(t_{0}\right)-\lambda D_{\nabla, t_{0}}^{\beta-j} y\left(t_{0}\right) \quad\left(j=l_{m-2}+1, \ldots, l_{m-1}\right), \\
d_{j}= & D_{\nabla, t_{0}}^{\alpha-j} y\left(t_{0}\right) \quad\left(j=l_{m-1}+1, \ldots, l\right) .
\end{aligned}
$$

Here, $\sum_{k=m}^{n} A_{k}:=0(m>n)$. For $z \in \mathbb{C}$ and $\left|\sum_{k=0}^{m-2} A_{k} z^{\alpha_{k}-\beta} /\left(z^{\alpha-\beta}-\lambda\right)\right|<1$, we have

$$
\begin{aligned}
& \frac{1}{z^{\alpha}-\lambda z^{\beta}-\sum_{k=0}^{m-2} A_{k} z^{\alpha_{k}}} \\
& =\frac{z^{-\beta}}{z^{\alpha-\beta}-\lambda} \cdot \frac{1}{\left(1-\left(\sum_{k=0}^{m-2} A_{k} z^{\alpha_{k}-\beta} /\left(z^{\alpha-\beta}-\lambda\right)\right)\right)} \\
& =\sum_{n=0}^{\infty} \frac{z^{-\beta}}{\left(z^{\alpha-\beta}-\lambda\right)^{n+1}}\left(\sum_{k=0}^{m-2} A_{k} z^{\alpha_{k}-\beta}\right)^{n} \\
& =\sum_{n=0}^{\infty}\left(\sum_{k_{0}+\cdots+k_{m-2}=n}\right) \frac{n !}{k_{0} ! \cdots k_{m-2} !} \\
& \times\left[\prod_{\nu=0}^{m-2}\left(A_{\nu}\right)^{k_{\nu}}\right] \frac{z^{-\beta-\sum_{\nu=0}^{m-2}\left(\beta-\alpha_{\nu}\right) k_{\nu}}}{\left(z^{\alpha-\beta}-\lambda\right)^{n+1}},
\end{aligned}
$$

if we also take into account the following relation:

$$
\begin{aligned}
\left(x_{0}+\cdots+x_{m-2}\right)^{n} & \\
& =\left(\sum_{k_{0}+\cdots+k_{m-2}=n}\right) \frac{n !}{k_{0} ! \cdots k_{m-2} !} \prod_{\nu=0}^{m-2} x_{v}^{k_{v}},
\end{aligned}
$$

where the summation is taken over all $k_{0}, \ldots, k_{m-2} \in \mathbb{N}_{0}$ such that $k_{0}+\cdots+k_{m-2}=n$.

In addition, for $z \in \mathbb{C}$ and $\left|\lambda z^{\beta-\alpha}\right|<1$, we have

$$
\begin{aligned}
& \frac{z^{j-1-\beta-\sum_{v=0}^{m-2}\left(\beta-\alpha_{\nu}\right) k_{\nu}}}{\left(z^{\alpha-\beta}-\lambda\right)^{n+1}} \\
& =\frac{z^{(\alpha-\beta)-\left(\alpha+1-j+\sum_{\nu=0}^{m-2}\left(\beta-\alpha_{\nu}\right) k_{\nu}\right)}}{\left(z^{\alpha-\beta}-\lambda\right)^{n+1}} \\
& =\frac{1}{n !} \mathscr{L}_{\nabla, t_{0}}\left\{\frac{\partial^{n}}{\partial \lambda^{n}} \nabla_{\alpha-\beta, \alpha+1-j+\sum_{\nu=0}^{m-2}\left(\beta-\alpha_{\nu}\right) k_{\nu}}\left(\lambda ; t, t_{0}\right)\right\}(z) .
\end{aligned}
$$

From (207), (209), and (211), we derive the solution to (205),

$$
y(t)=\sum_{j=1}^{l} d_{j} y_{j}(t)
$$


which shows that arbitrary solution $y(t)$ can be expressed by $y_{j}(t), j=1, \ldots, l$, where $y_{j}(t)(j=1, \ldots, l)$ are given by (206). For $q, j=1, \ldots, l$, the direct evaluation yields

$$
\begin{aligned}
& D_{\nabla, t_{0}}^{\alpha-q} y_{j}(t) \\
& =D_{\nabla, t_{0}}^{\alpha-q}\left\{\sum_{n=0}^{\infty}\left(\sum_{k_{0}+\cdots+k_{m-2}=n}\right) \frac{1}{k_{0} ! \cdots k_{m-2} !}\right. \\
& \times\left[\prod_{v=0}^{m-2}\left(A_{\nu}\right)^{k_{v}}\right] \\
& \left.\times \frac{\partial^{n}}{\partial \lambda^{n}} \nabla F_{\alpha-\beta, \alpha+1-j+\sum_{\nu=0}^{m-2}\left(\beta-\alpha_{\nu}\right) k_{\nu}}\left(\lambda ; t, t_{0}\right)\right\} \\
& =\sum_{n=0}^{\infty}\left(\sum_{k_{0}+\cdots+k_{m-2}=n}\right) \frac{1}{k_{0} ! \cdots k_{m-2} !} \\
& \times\left[\prod_{v=0}^{m-2}\left(A_{\nu}\right)^{k_{v}}\right] \\
& \times\left\{\frac{\partial^{n}}{\partial \lambda^{n}} D_{\nabla, t_{0}}^{\alpha-q}\left[\sum_{s=0}^{\infty} \lambda^{s} \widehat{h}_{s(\alpha-\beta)+\alpha-j+\sum_{\nu=0}^{m-2}\left(\beta-\alpha_{\nu}\right) k_{\nu}}\left(t, t_{0}\right)\right]\right\} \\
& =\sum_{n=0}^{\infty}\left(\sum_{k_{0}+\cdots+k_{m-2}=n}\right) \frac{1}{k_{0} ! \cdots k_{m-2} !} \\
& \times\left[\prod_{v=0}^{m-2}\left(A_{v}\right)^{k_{v}}\right] \\
& \times\left\{\frac{\partial^{n}}{\partial \lambda^{n}} \sum_{s=0}^{\infty} \lambda^{s} \widehat{h}_{s(\alpha-\beta)+\sum_{\nu=0}^{m-2}\left(\beta-\alpha_{\nu}\right) k_{\nu}+q-j}\left(t, t_{0}\right)\right\} \text {. }
\end{aligned}
$$

For $q>j, D_{\nabla, t_{0}}^{\alpha-q} y_{j}\left(t_{0}\right)=0$, and for $q=j, D_{\nabla, t_{0}}^{\alpha-q} y_{j}\left(t_{0}\right)=1$. Thus, we have $W_{\alpha}\left(t_{0}\right)=1$. It follows from Lemma 53 that the functions $y_{j}(t), j=1,2, \ldots, l$ in (206) are linearly independent solutions and then they yield the fundamental system of solutions to (205). Furthermore, if $q<j$, then we rewrite (213) as follows:

$$
\begin{aligned}
D_{\nabla, t_{0}}^{\alpha-q} y_{j} & (t) \\
= & D_{\nabla, t_{0}}^{\alpha-q} \widehat{h}_{\alpha-j}\left(t, t_{0}\right) \\
& +\sum_{s=1}^{\infty} \lambda^{s} \widehat{h}_{s(\alpha-\beta)+\sum_{\nu=0}^{m-2}\left(\beta-\alpha_{\nu}\right) k_{\nu}+q-j}\left(t, t_{0}\right) \\
& +\sum_{n=1}^{\infty}\left(\sum_{k_{0}+\cdots+k_{m-2}=n}\right) \frac{1}{k_{0} ! \cdots k_{m-2} !} \\
& \times\left[\prod_{\nu=0}^{m-2}\left(A_{\nu}\right)^{k_{\nu}}\right] \frac{\partial^{n}}{\partial \lambda^{n}} \widehat{h}_{\sum_{\nu=0}^{m-2}\left(\beta-\alpha_{\nu}\right) k_{\nu}+q-j}\left(t, t_{0}\right)
\end{aligned}
$$

$$
\begin{aligned}
& +\sum_{n=1}^{\infty}\left(\sum_{k_{0}+\cdots+k_{m-2}=n}\right) \frac{1}{k_{0} ! \cdots k_{m-2} !} \\
& \times\left[\prod_{\nu=0}^{m-2}\left(A_{\nu}\right)^{k_{\nu}}\right] \\
& \times\left\{\frac{\partial^{n}}{\partial \lambda^{n}} \sum_{s=1}^{\infty} \lambda^{s} \widehat{h}_{s(\alpha-\beta)+\sum_{\nu=0}^{m-2}\left(\beta-\alpha_{\nu}\right) k_{\nu}+q-j}\left(t, t_{0}\right)\right\} \\
& =\sum_{s=1}^{\infty} \lambda^{s} \widehat{h}_{s(\alpha-\beta)+q-j}\left(t, t_{0}\right) \\
& +\sum_{n=1}^{\infty}\left(\sum_{k_{0}+\cdots+k_{m-2}=n}\right) \frac{1}{k_{0} ! \cdots k_{m-2} !} \\
& \times\left[\prod_{\nu=0}^{m-2}\left(A_{\nu}\right)^{k_{\nu}}\right] \frac{\partial^{n}}{\partial \lambda^{n}} \widehat{h}_{\sum_{\nu=0}^{m-2}\left(\beta-\alpha_{v}\right) k_{\nu}+q-j}\left(t, t_{0}\right) \\
& +\sum_{n=1}^{\infty}\left(\sum_{k_{0}+\cdots+k_{m-2}=n}\right) \\
& \times \frac{1}{k_{0} ! \cdots k_{m-2} !}\left[\prod_{\nu=0}^{m-2}\left(A_{\nu}\right)^{k_{\nu}}\right] \\
& \times\left\{\frac{\partial^{n}}{\partial \lambda^{n}} \sum_{s=1}^{\infty} \lambda^{s} \widehat{h}_{s(\alpha-\beta)+\sum_{\nu=0}^{m-2}\left(\beta-\alpha_{\nu}\right) k_{\nu}+q-j}\left(t, t_{0}\right)\right\} \text {. }
\end{aligned}
$$

If $\alpha+1-l>\beta>\alpha_{m-2}+l-1$, then $s(\alpha-\beta)+q-j \geq$ $(\alpha-\beta)+1-l>0$ for $n=0, q, j=1, \ldots, l, s \in \mathbb{N}^{+}$, and $\sum_{\nu=0}^{m-2}\left(\beta-\alpha_{v}\right) k_{v}+q-j \geq \beta-\alpha_{i}+1-l \geq \beta-\alpha_{m-2}+1-l>0$ for $s=0, q, j=1, \ldots, l, n \in \mathbb{N}^{+}$. Besides, we also have $s(\alpha-$ $\beta)+\sum_{\nu=0}^{m-2}\left(\beta-\alpha_{v}\right) k_{v}+q-j \geq(\alpha-\beta)+\beta-\alpha_{i}+1-l \geq$ $(\alpha-\beta)+\beta-\alpha_{m-2}+1-l>0$ for $q, j=1, \ldots, l, s, n \in \mathbb{N}^{+}$. These imply that $D_{\nabla, t_{0}}^{\alpha-q} y_{j}\left(t_{0}\right)=0$. Thus the relations in (180) are valid. The result follows.

\section{Nonhomogeneous Equations with Constant Coefficients}

In above section, we applied the Laplace transform method to derive explicit solutions to the homogeneous equations (169) with the Riemann-Liouville fractional derivatives. Here, we use this approach to find particular solutions to the corresponding nonhomogeneous equations as follows:

$$
\begin{gathered}
\sum_{k=1}^{m} A_{k} D_{\nabla, t_{0}}^{\alpha_{k}} y(t)+A_{0} y(t)=f(t) \\
\left(t>t_{0} ; 0<\alpha_{1}<\cdots<\alpha_{m} ; m \in \mathbb{N}\right),
\end{gathered}
$$

with real $A_{k} \in \mathbb{R}(k=0, \ldots, m)$.

By (170) and (171), for suitable functions $y$, the Laplace transform of $D_{\nabla, t_{0}}^{\alpha} y$ is given by

$$
\mathscr{L}_{\nabla, t_{0}}\left\{D_{\nabla, t_{0}}^{\alpha} y(t)\right\}(z)=z^{\alpha} \mathscr{L}_{\nabla, t_{0}}\{y(t)\}(z) .
$$


Applying the Laplace transform to (215) and taking (216) into account, we have

$$
\left[A_{0}+\sum_{k=1}^{m} A_{k} z^{\alpha_{k}}\right] \mathscr{L}_{\nabla, t_{0}}\{y(t)\}(z)=\mathscr{L}_{\nabla, t_{0}}\{f(t)\}(z) .
$$

Using the inverse Laplace transform $\mathscr{L}_{\nabla, t_{0}}^{-1}$ from here we obtain a particular solution to (215) in the following form:

$$
y(t)=\mathscr{L}_{\nabla, t_{0}}^{-1}\left[\frac{\mathscr{L}_{\nabla, t_{0}}\{f(t)\}(z)}{A_{0}+\sum_{k=1}^{m} A_{k} z^{\alpha_{k}}}\right](t)
$$

Using the Laplace convolution formula

$$
\mathscr{L}_{\nabla, t_{0}}\{f * g\}(z)=\mathscr{L}_{\nabla, t_{0}}\{f\}(z) \mathscr{L}_{\nabla, t_{0}}\{g\}(z),
$$

we can introduce the Laplace fractional analog of the Green function as follows:

$$
\begin{aligned}
& G_{\alpha_{1}, \ldots, \alpha_{m}}(t)=\mathscr{L}_{\nabla, t_{0}}^{-1}\left\{\frac{1}{P_{\alpha_{1}, \ldots, \alpha_{m}}(z)}\right\}(t), \\
& P_{\alpha_{1}, \ldots, \alpha_{m}}(z)=A_{0}+\sum_{k=1}^{m} A_{k} z^{\alpha_{k}}
\end{aligned}
$$

and express a particular solution of (181) in the form of the Laplace convolution $G_{\alpha_{1}, \ldots, \alpha_{m}}(t)$ and $f(t)$ as follows:

$$
y(t)=G_{\alpha_{1}, \ldots, \alpha_{m}}(t) * f(t) .
$$

Generally speaking, we can consider (215) with $A_{m}=1$. First, we derive a particular solution to (215) with $m=1$ in the following form:

$$
D_{\nabla, t_{0}}^{\alpha} y(t)-\lambda y(t)=f(t) \quad\left(t>t_{0} ; \alpha>0\right) .
$$

Theorem 59. Let $\alpha>0, \lambda \in \mathbb{R}$. Then, (222) is solvable, and its particular solution has the following form:

$$
y(t)={ }_{\nabla} F_{\alpha, \alpha}\left(\lambda ; t, t_{0}\right) * f(t) .
$$

Proof. Equation (222) is (215) with $m=1, \alpha_{1}=\alpha, A_{1}=1$, $A_{0}=-\lambda$ and (220) takes the following form:

$$
G_{\alpha}(t)=\mathscr{L}_{\nabla, t_{0}}^{-1}\left\{\frac{1}{z^{\alpha}-\lambda}\right\}(t)={ }_{\nabla} F_{\alpha, \alpha}\left(\lambda ; t, t_{0}\right) .
$$

Thus, (221), with $G_{\alpha_{1}, \ldots, \alpha_{m}}(t)=G_{\alpha}(t)$, yields (223). The result is proved.

Next, we derive a particular solution to (215) with $m=2$ of the following form:

$$
\begin{gathered}
D_{\nabla, t_{0}}^{\alpha} y(t)-\lambda D_{\nabla, t_{0}}^{\beta} y(t)-\mu y(t)=f(t) \\
\left(t>t_{0} ; \alpha>\beta>0\right) .
\end{gathered}
$$

Theorem 60. Let $\alpha>\beta>0, \lambda, \mu \in \mathbb{R}$. Then, (225) is solvable, and its particular solution has the following form:

$$
\begin{gathered}
y(t)=G_{\alpha, \beta ; \lambda, \mu}(t) * f(t), \\
G_{\alpha, \beta ; \lambda, \mu}(t)=\sum_{k=0}^{\infty} \frac{\mu^{k}}{k !} \frac{\partial^{k}}{\partial \lambda^{k}}{ }_{\nabla} F_{\alpha-\beta, \alpha+k \beta}\left(\lambda ; t, t_{0}\right),
\end{gathered}
$$

provided that the series in (227) is convergent.

Proof. Equation (225) is the same as (215) with $m=2, \alpha_{2}=\alpha$, $\alpha_{1}=\beta, A_{2}=1, A_{1}=-\lambda, A_{0}=-\mu$, and (220) is given by

$$
G_{\alpha, \beta ; \lambda, \mu}(t)=\mathscr{L}_{\nabla, t_{0}}^{-1}\left\{\frac{1}{z^{\alpha}-\lambda z^{\beta}-\mu}\right\}(t) .
$$

According to (197) for $z \in \mathbb{C}$ and $\left|\mu z^{-\beta} /\left(z^{\alpha-\beta}-\lambda\right)\right|<1$,we have

$$
G_{\alpha, \beta ; \lambda, \mu}(t)=\mathscr{L}_{\nabla, t_{0}}^{-1}\left\{\sum_{n=0}^{\infty} \mu^{n} \frac{z^{-\beta-n \beta}}{\left(z^{\alpha-\beta}-\lambda\right)^{n+1}}\right\}(t) .
$$

In addition, for $z \in \mathbb{C}$ and $\left|\lambda z^{\beta-\alpha}\right|<1$, we have

$$
\frac{z^{-\beta-n \beta}}{\left(z^{\alpha-\beta}-\lambda\right)^{n+1}}=\frac{1}{n !} \mathscr{L}_{\nabla, t_{0}}\left\{\frac{\partial^{n}}{\partial \lambda^{n}{ }_{\nabla} F_{\alpha-\beta, \alpha+n \beta}}\left(\lambda ; t, t_{0}\right)\right\}(z),
$$

and hence (229) takes the following form:

$$
G_{\alpha, \beta ; \lambda, \mu}(t)=\sum_{n=0}^{\infty} \frac{\mu^{n}}{n !} \frac{\partial^{n}}{\partial \lambda^{n}} \nabla_{\alpha-\beta, \alpha+n \beta}\left(\lambda ; t, t_{0}\right) .
$$

Thus, the result in (226) follows from (221) with $G_{\alpha_{1}, \ldots, \alpha_{m}}(t)=$ $G_{\alpha, \beta ; \lambda, \mu}(t)$.

Finally, we find a particular solution to (215) with any $m \in$ $\mathbb{N} \backslash\{1,2\}$. It is convenient to rewrite (215), just as (205) in the following form:

$$
D_{\nabla, t_{0}}^{\alpha} y(t)-\lambda D_{\nabla, t_{0}}^{\beta} y(t)-\sum_{k=1}^{m-2} A_{k} D_{\nabla, t_{0}}^{\alpha_{k}} y(t)-A_{0} y(t)=f(t)
$$

with $m \in \mathbb{N} \backslash\{1,2\}, 0<\alpha_{1}<\cdots<\alpha_{m-2}<\beta<\alpha$, and $\lambda, A_{0}, \ldots, A_{m-2} \in \mathbb{R}$.

Theorem 61. Let $m \in \mathbb{N} \backslash\{1,2\}, 0=\alpha_{0}<\alpha_{1}<\cdots<\alpha_{m-2}<$ $\beta<\alpha$, and let $\lambda, A_{0}, \ldots, A_{m-2} \in \mathbb{R}$. Then, (232) is solvable, and its particular solution has the following form:

$$
y(t)=G_{\alpha_{1}, \ldots, \alpha_{m-2}, \beta, \alpha ; \lambda}(t) * f(t),
$$

$$
\begin{aligned}
& G_{\alpha_{1}, \ldots, \alpha_{m-2}, \beta, \alpha ; \lambda}(t) \\
& =\sum_{n=0}^{\infty}\left(\sum_{k_{0}+\cdots+k_{m-2}=n}\right) \frac{1}{k_{0} ! \cdots k_{m-2} !}\left[\prod_{\nu=0}^{m-2}\left(A_{\nu}\right)^{k_{\nu}}\right] \\
& \quad \times \frac{\partial^{n}}{\partial \lambda^{n}} \nabla F_{\alpha-\beta, \alpha+\sum_{\nu=0}^{m-2}\left(\beta-\alpha_{\nu}\right) k_{\nu}}\left(\lambda ; t, t_{0}\right),
\end{aligned}
$$


provided that the series (234) is convergent. The inner sum is taken over all $k_{0}, \ldots, k_{m-2}$ such that $k_{0}+\cdots+k_{m-2}=n$.

Proof. Equation (232) is the same equation as (215) with $\alpha_{m}=$ $\alpha, \alpha_{m-1}=\beta, A_{m}=1, \ldots, A_{m-1}=-\lambda$, and with $-A_{k}$ instead of $A_{k}$ for $k=0, \ldots, m-2$. Since $\alpha_{0}=0$, (220) takes the following form:

$$
G_{\alpha_{1}, \ldots, \alpha_{m-2}, \beta, \alpha ; \lambda}(t)=\mathscr{L}_{\nabla, t_{0}}^{-1}\left\{\frac{1}{z^{\alpha}-\lambda \alpha^{\beta}-\sum_{k=0}^{m-2} A_{k} z^{\alpha_{k}}}\right\}(t) .
$$

For $z \in \mathbb{C}$ and $\left|\sum_{k=0}^{m-2} A_{k} z^{\alpha_{k}-\beta} /\left(z^{\alpha-\beta}-\lambda\right)\right|<1$, in accordance with (209), we have

$$
\begin{aligned}
G_{\alpha_{1}, \ldots, \alpha_{m-2}, \beta, \alpha ; \lambda} & (t) \\
=\mathscr{L}_{\nabla, t_{0}}^{-1} & \left\{\sum_{n=0}^{\infty}\left(\sum_{k_{0}+\cdots+k_{m-2}=n}\right) \frac{n !}{k_{0} ! \cdots k_{m-2} !}\right. \\
& \left.\times\left[\prod_{\nu=0}^{m-2}\left(A_{\nu}\right)^{k_{\nu}}\right] \frac{z^{-\beta-\sum_{\nu=0}^{m-2}\left(\beta-\alpha_{\nu}\right) k_{\nu}}}{\left(z^{\alpha-\beta}-\lambda\right)^{n+1}}\right\}(t) .
\end{aligned}
$$

For $z \in \mathbb{C}$ and $\left|\lambda z^{\beta-\alpha}\right|<1$, we have

$$
\begin{aligned}
& \frac{z^{-\beta-\sum_{v=0}^{m-2}\left(\beta-\alpha_{\nu}\right) k_{v}}}{\left(z^{\alpha-\beta}-\lambda\right)^{n+1}} \\
& \quad=\frac{1}{n !} \mathscr{L}_{\nabla, t_{0}}\left\{\frac{\partial^{n}}{\partial \lambda^{n}} \nabla F_{\alpha-\beta, \alpha+\sum_{\nu=0}^{m-2}\left(\beta-\alpha_{v}\right) k_{v}}\left(\lambda ; t, t_{0}\right)\right\}(z) .
\end{aligned}
$$

The proof is finished.

As in the case of ordinary differential equations, a general solution to the nonhomogeneous equation (215) is a sum of a particular solution to this equation and of the general solution to the corresponding homogeneous equation (169). Therefore, the results established in this section and in the previous section can be used to derive general solutions to the nonhomogeneous equation (222), (225), and (232). The following statements can thus be derived from Theorems 54, 59, $56,60,58$, and 61 , respectively.

Theorem 62. Let $l-1<\alpha \leq l(l \in \mathbb{N}), \lambda \in \mathbb{R}$. Then, (225) is solvable, and its general solution is given by

$$
y(t)=\sum_{j=1}^{l} c_{j \nabla} F_{\alpha, \alpha+1-j}\left(\lambda ; t, t_{0}\right)+{ }_{\nabla} F_{\alpha, \alpha}\left(\lambda ; t, t_{0}\right) * f(t),
$$

where $c_{j}(j=1, \ldots, l)$ are arbitrary real constants.
Theorem 63. Let $l-1<\alpha \leq l(l \in \mathbb{N}), 0<\beta<\alpha, \lambda, \mu \in$ $\mathbb{R}$. Then, (225) is solvable, and its general solution has the following form:

$$
\begin{aligned}
y(t)= & \sum_{j=1}^{l} c_{j} \sum_{n=0}^{\infty} \frac{\mu^{n}}{n !} \frac{\partial^{n}}{\partial \lambda^{n}} \nabla F_{\alpha-\beta, \alpha+n \beta+1-j}\left(\lambda ; t, t_{0}\right) \\
& +G_{\alpha, \beta ; \lambda, \mu}(t) * f(t),
\end{aligned}
$$

where $G_{\alpha, \beta, \lambda, \mu}(t)$ is given by $(227)$ and $c_{j}(j=1, \ldots, l)$ are arbitrary real constants.

Theorem 64. Let $m \in \mathbb{N} \backslash\{1,2\}, l-1<\alpha \leq l(l \in \mathbb{N})$ and let $\beta$ and $\alpha_{1}, \alpha_{2}, \ldots, \alpha_{m-2}$ be such that $\alpha>\beta>\alpha_{m-2}>\cdots>\alpha_{1}>$ $\alpha_{0}=0$ and $\alpha-l+1 \geq \beta$ and let $\lambda, A_{0}, \ldots, A_{m-2} \in \mathbb{R}$. Then, (232) is solvable, and its general solution is given by

$$
\begin{aligned}
y(t)= & \sum_{j=1}^{l} c_{j} \sum_{n=0}^{\infty}\left(\sum_{k_{0}+\cdots+k_{m-2}=n}\right) \frac{1}{k_{0} ! \cdots k_{m-2} !} \\
& \times\left[\prod_{\nu=0}^{m-2}\left(A_{\nu}\right)^{k_{\nu}}\right] \\
& \times \frac{\partial^{n}}{\partial \lambda^{n}} F_{\alpha-\beta, \alpha+1-j+\sum_{\nu=0}^{m-2}\left(\beta-\alpha_{\nu}\right) k_{\nu}}\left(\lambda ; t, t_{0}\right) \\
& +G_{\alpha_{1}, \ldots, \alpha_{m-2}, \beta, \alpha ; \lambda}(t) * f(t),
\end{aligned}
$$

where $G_{\alpha_{1}, \ldots, \alpha_{m-2, \beta, \alpha ; \lambda}}(t)$ is given by $(234)$ and $c_{j}(j=1, \ldots, l)$ are arbitrary real constants.

\section{Acknowledgments}

First, the authors are very grateful to the referees for their careful reading of the paper, and a lot of valuable comments, which greatly improved this manuscript. Next, This work was supported by the National Natural Science Foundation of China (11171286), and by the Jiangsu Province Colleges and Universities Graduate Scientific Research Innovative Program (CXZZ12-0974).

\section{References}

[1] A. A. Kilbas, H. M. Srivastava, and J. J. Trujillo, Theory and Applications of Fractional Differential Equations, vol. 204 of NorthHolland Mathematics Studies, Elsevier, London, UK, 2006.

[2] M. Bohner and A. Peterson, Advances in Dynamic Equations on Time Scales, Birkhäuser, Boston, Mass, USA, 2003, Edited by Martin Bohner and Allan Peterson.

[3] M. Bohner and A. Peterson, Dynamic Equations on Time Scales, Birkhäuser, Boston, Mass, USA, 2001, An introduction with applications.

[4] M. Bohner and G. Sh. Guseinov, "Partial differentiation on time scales," Dynamic Systems and Applications, vol. 13, no. 3-4, pp. 351-379, 2004.

[5] G. A. Anastassiou, "Foundations of nabla fractional calculus on time scales and inequalities," Computers \& Mathematics with Applications, vol. 59, no. 12, pp. 3750-3762, 2010. 
[6] P. A. Williams, Unifying fractional calculus with time scales [doctoral thesis], The University of Melbourne, 2012.

[7] N. R. D. O. Bastos, Fractional calculus on time scales [doctoral thesis], The University of Aveiro, 2012.

[8] J. Cheng, Theory of Fractional Difference Equation, Xiamen University Press, 2011.

[9] A. Denı, Measure Theory on Time Scales, 1zmır, 2007.

[10] J. M. Davis, I. A. Gravagne, B. J. Jackson, R. J. Marks, II, and A. A. Ramos, "The Laplace transform on time scales revisited," Journal of Mathematical Analysis and Applications, vol. 332, no. 2, pp. 1291-1307, 2007.

[11] M. Bohner and G. Sh. Guseinov, "The convolution on time scales," Abstract and Applied Analysis, vol. 2007, Article ID 58373, 24 pages, 2007. 


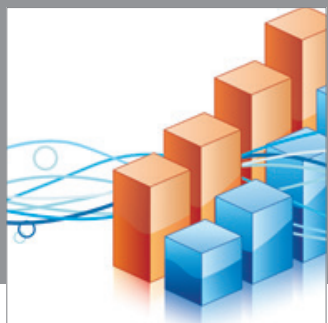

Advances in

Operations Research

mansans

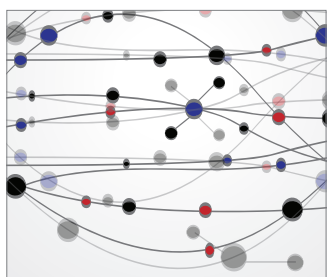

The Scientific World Journal
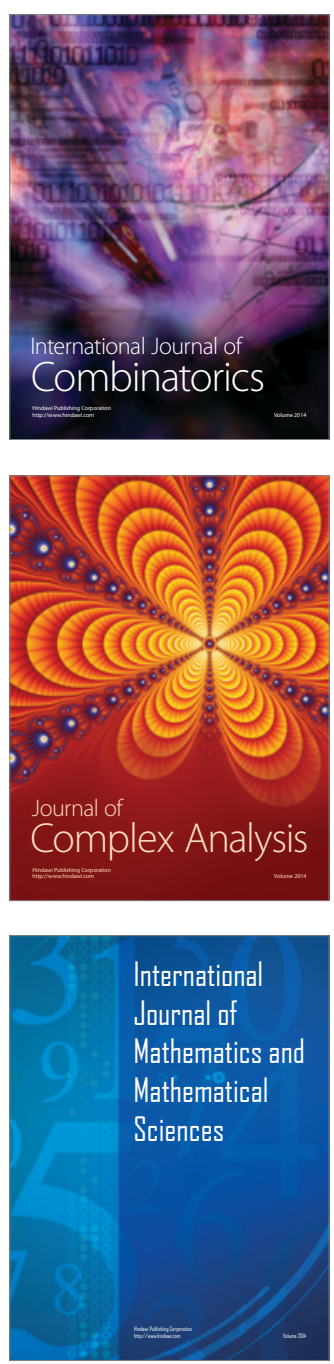
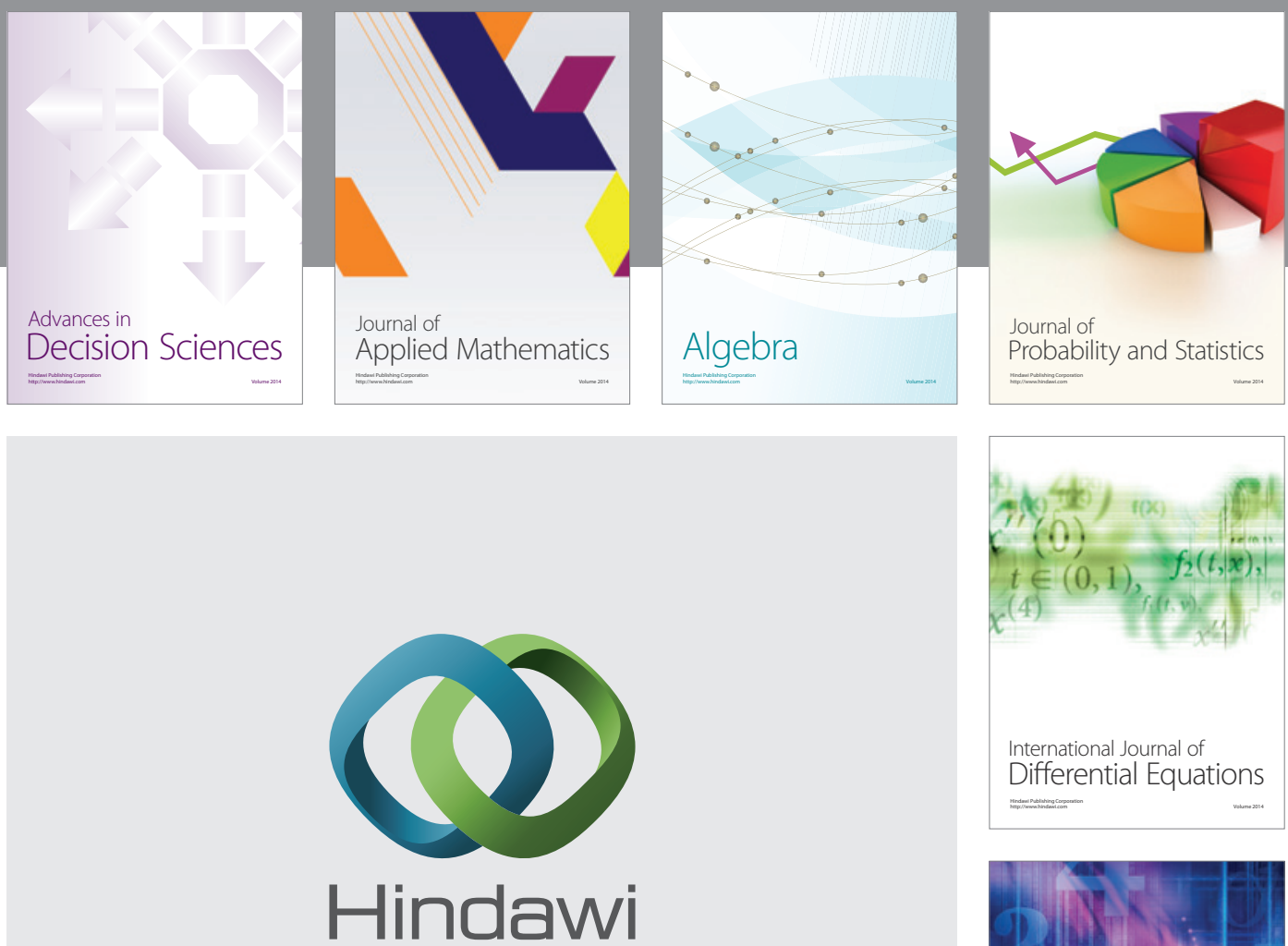

Submit your manuscripts at http://www.hindawi.com
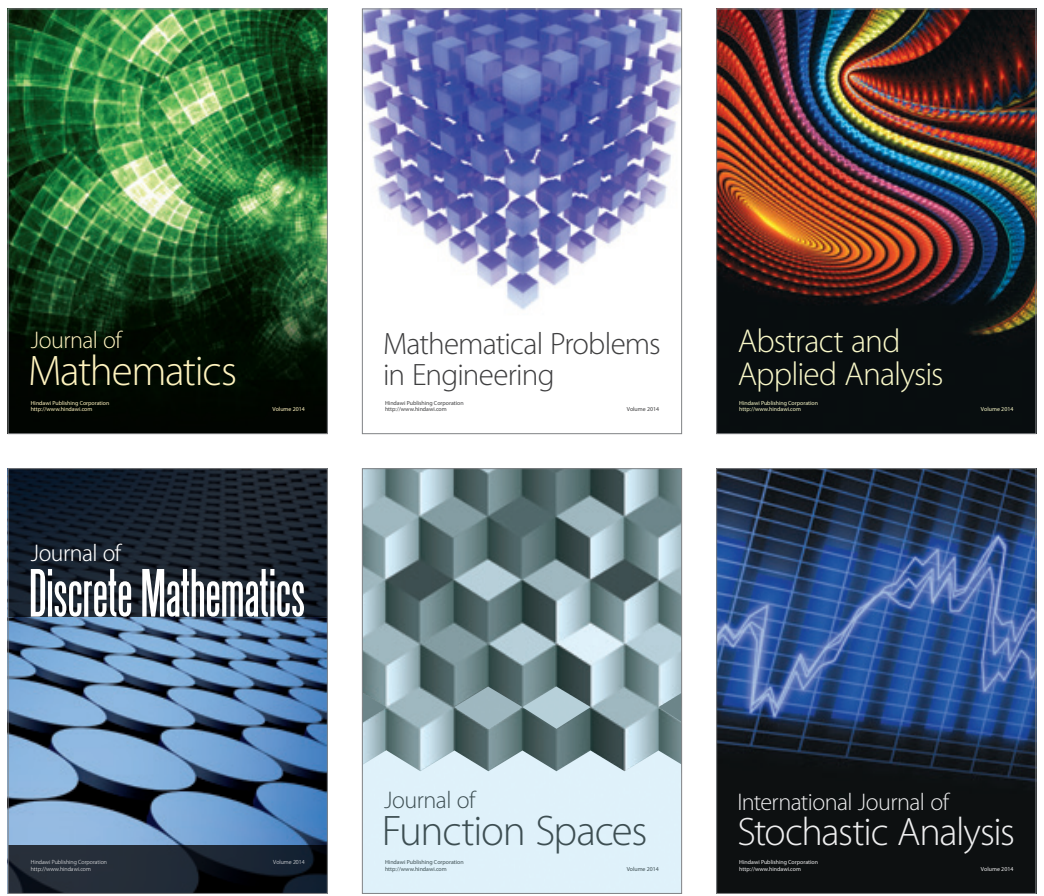

Journal of

Function Spaces

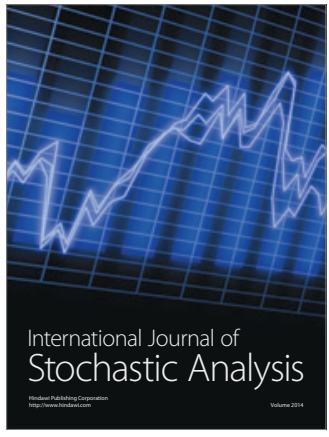

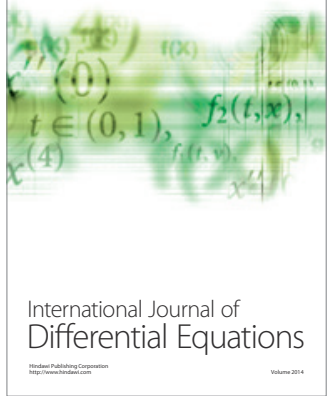
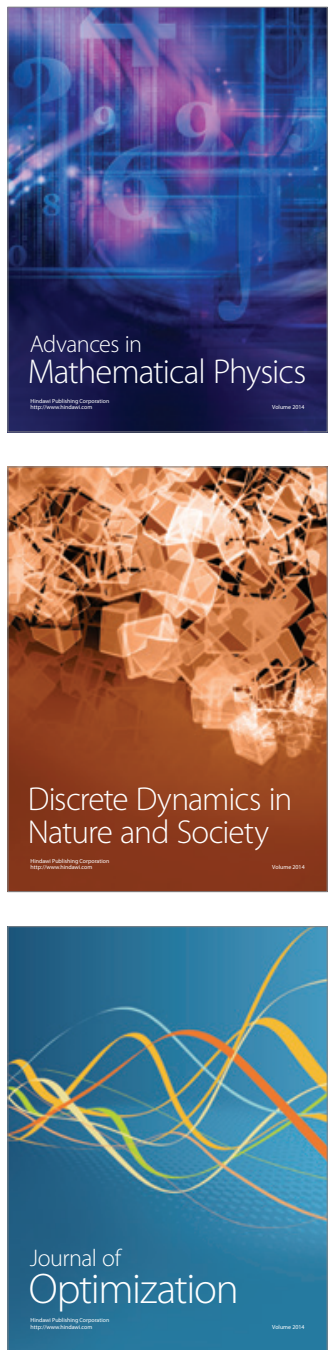